\title{
A Perspective on Late-Stage Aromatic C-H Bond Functionalization
}

Li Zhang and Tobias Ritter*

Max-Planck-Institute für Kohlenforschung, Kaiser-Wilhelm Platz 1, D-45470 Mülheim an der Ruhr, Germany.

*E-mail: ritter@kofo.mpg.de 


\section{TABLE OF CONTENTS}

TABLE OF CONTENTS

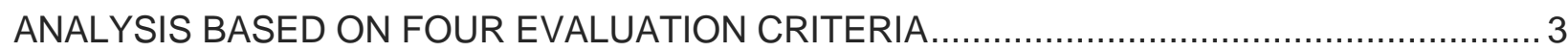

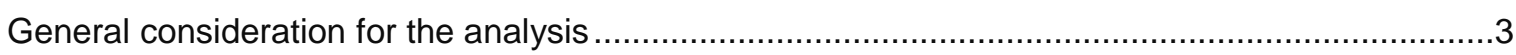

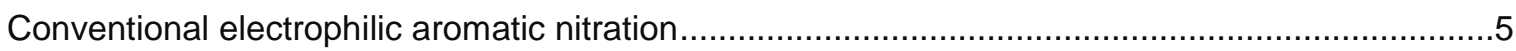

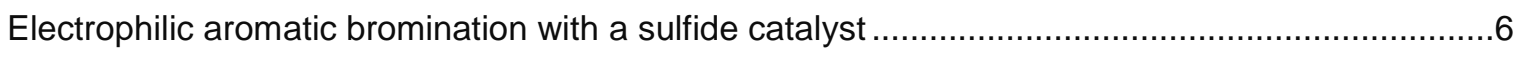

Electrophilic aromatic fluorination via a [PdIV]-F species.....................................................

Electrophilic aromatic oxygenation with bis(methanesulfonyl) peroxide .......................................

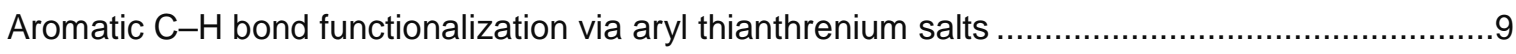

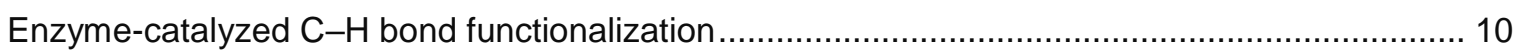

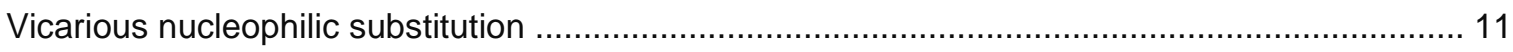

Aromatic $\mathrm{C}-\mathrm{H}$ bond functionalization via pyridyl phosphonium salts .......................................... 12

Functionalization of electron-rich (het)arenes via electrophilic metalation .................................. 13

Palladium-catalyzed non-directed $\mathrm{C}-\mathrm{H}$ bond olefination enabled by ligand ................................ 14

Palladium-catalyzed non-directed $\mathrm{C}-\mathrm{H}$ bond cyanation enabled by ligand ................................... 15

Palladium-catalyzed ortho $\mathrm{C}-\mathrm{H}$ bond hydroxylation of aryl carboxylic acids ............................... 16

Palladium-catalyzed remote $\mathrm{C}-\mathrm{H}$ bond olefination of quinoline via template ............................... 17

Palladium-catalyzed remote $\mathrm{C}-\mathrm{H}$ bond arylation of quinoline via template and norbornene ......... 18

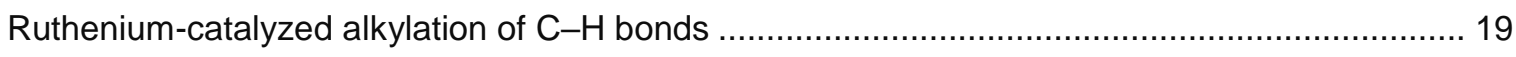

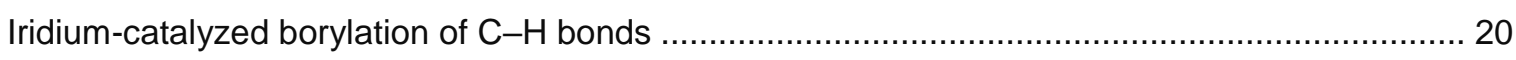

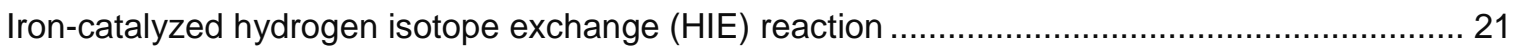

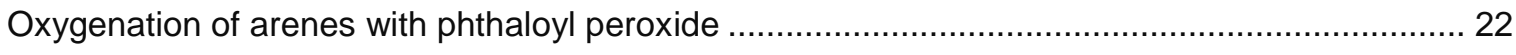

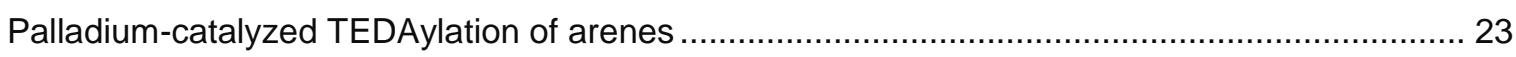

Electrochemical trifluoromethylation of hetarene with zinc sulfinate reagents.............................. 24

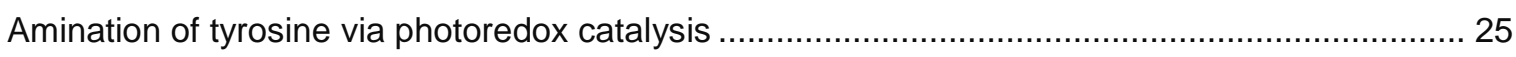

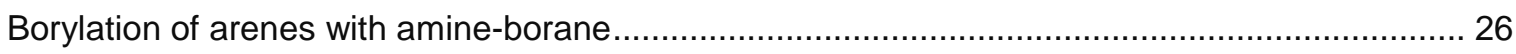

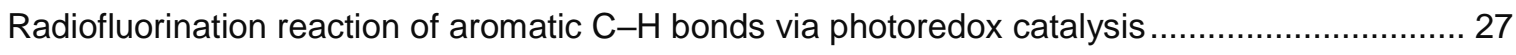

Hydroxylation of aromatic $\mathrm{C}-\mathrm{H}$ bonds via electrophotoredox catalysis ...................................... 28

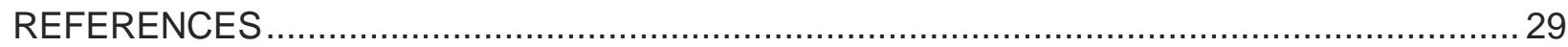




\section{ANALYSIS BASED ON FOUR EVALUATION CRITERIA}

\section{General consideration for the analysis}

The evaluation of reactivity, chemoselectivity, site-selectivity, and substrate scope for the selected reactions would best be done by an objective, quantitative, and comparative analysis. Unfortunately, such a quantitative analysis is not possible at the current stage because the reactions that we have evaluated have not been conducted under identical conditions, or even with the same substrates. In addition, evaluation based on specific simple substrates for which data may be available for several reactions such as anisole, aniline or related compounds are not very informative when it comes to late-stage functionalization potential. Analysis of representative observables as discussed below served as a basis for a semi-quantitative scoring that can give a vague characterization of the reactions' attributes. It cannot serve as a quantitative comparison between reactions but provide an analysis of the remaining challenges of the field. This data, in our opinion, is more valuable than not providing any scoring system albeit subjective and prone to disagreement and error.

\begin{tabular}{|c|c|c|}
\hline Evaluation criteria & Score & Description \\
\hline \multirow{4}{*}{ Reactivity } & Low & $\begin{array}{l}\text { Reaction temperature higher than } 120^{\circ} \mathrm{C} \text { or otherwise harsh } \\
\text { reaction conditions are required for most substrates, and the } \\
\text { reaction usually shows less than } 50 \% \text { conversion or longer than } \\
3 \text { days reaction time on many substrates. }\end{array}$ \\
\hline & Moderate & $\begin{array}{l}\text { Reaction temperature higher than } 50^{\circ} \mathrm{C} \text { are required to achive more } \\
\text { than } 50 \% \text { conversion for most substrates, or the reaction time is } \\
\text { longer than } 24 \mathrm{~h} \text {. For a catalyzed reaction, the conversion is less } \\
\text { than } 50 \% \text { at } 1-5 \text { mol\% catalyst loading but synthetically useful } \\
\text { yields can be obtained when the catalyst loading is increased to 5- } \\
20 \mathrm{~mol} \% \text {. }\end{array}$ \\
\hline & High & $\begin{array}{l}\text { More than } 90 \% \text { conversion can be achieved for the majority of } \\
\text { substrates that participate successfully in the reaction. Reaction } \\
\text { temperature is below } 50^{\circ} \mathrm{C} \text { for most substrates. For a catalyzed } \\
\text { reaction, the catalyst should have a turnover number of at least } 20 \text {. }\end{array}$ \\
\hline & Excellent & $\begin{array}{l}\text { Most substrates that proceed successfully in the reaction are } \\
\text { converted to more than } 95 \% \text { conversion at } 25^{\circ} \mathrm{C} \text { or below in less } \\
\text { than } 1 \mathrm{~h} \text {. For a catalyzed reaction, the loading of catalyst should be } \\
\text { at } 1 \mathrm{~mol} \% \text { or below. }\end{array}$ \\
\hline
\end{tabular}




\begin{tabular}{|c|c|c|}
\hline \multirow{4}{*}{ Chemoselectivity } & Low & $\begin{array}{l}\text { The majority of functional groups other than the arene interfere with } \\
\text { the reaction and cannot be tolerated. The reaction cannot tolerate } \\
\text { other reactive } \mathrm{C}-\mathrm{H} \text { bonds, such as benzylic or allylic } \mathrm{C}-\mathrm{H} \text { bonds. }\end{array}$ \\
\hline & Moderate & $\begin{array}{l}\text { Most other } \mathrm{C}-\mathrm{H} \text { bonds are tolerated. The reaction tolerates a variety } \\
\text { of functional groups but may not tolerate amines, alcohols, } \\
\text { carboxylic acids, basic heterocycles, sulfides or other fuctional } \\
\text { groups that have high reactivity with electrophilic, nucleophilic, or } \\
\text { Lewis acidic and -basic reagents or catalysts. }\end{array}$ \\
\hline & High & $\begin{array}{l}\text { In addition to most functional groups, highly reactive functional } \\
\text { groups such as amines, aldehydes, or carboxylic acids are tolerated } \\
\text { to some extent, and reactions can be carried out without protecting } \\
\text { groups on such functional groups. Additonally, several complex } \\
\text { small molecules can be functionalized. }\end{array}$ \\
\hline & Excellent & $\begin{array}{l}\text { Various complex molecules with miscellaneous functional groups } \\
\text { can be functionalized chemoselectively at the arene } \mathrm{C}-\mathrm{H} \text { bonds. }\end{array}$ \\
\hline \multirow{4}{*}{ Site-selectivity } & Low & $\begin{array}{l}\text { Undesired constitutional isomers or functionalization at more than } \\
\text { one site with a ratio of smaller than } 4: 1 \text { (desired constitutional } \\
\text { isomer : all other isomers) are observed for most substrates. }\end{array}$ \\
\hline & Moderate & $\begin{array}{l}\text { Undesired constitutional isomers or functionalization at more than } \\
\text { one site with a ratio of higher than } 4: 1 \text { but smaller than } 10: 1 \\
\text { (desired constitutional isomer : all other isomers) are observed for } \\
\text { several substrates. }\end{array}$ \\
\hline & High & $\begin{array}{l}\text { Undesired constitutional isomers or functionalization at more than } \\
\text { one site with a ratio of higher than } 10: 1 \text { but smaller than } 50: 1 \\
\text { (desired constitutional isomer : all other isomers) are observed for } \\
\text { most substrates. }\end{array}$ \\
\hline & Excellent & $\begin{array}{l}\text { Undesired constitutional isomers or functionalization at more than } \\
\text { one site are not observed for most substrates. The desired product } \\
\text { is produced in a ratio of larger than } 50: 1 \text { (desired constitutional } \\
\text { isomer : all other isomers). }\end{array}$ \\
\hline \multirow{4}{*}{ Substrate scope } & Low & $\begin{array}{l}\text { The reaction can only functionalize a few specific types of } \\
\text { substrates with a specific, required functional group, or substitution } \\
\text { pattern, such as a transformation that can only functionalize } \\
\text { nitrobenzenes, or a reaction that only works on 1,4-disubstituted } \\
\text { benzenes. }\end{array}$ \\
\hline & Moderate & $\begin{array}{l}\text { One category of arene substrates can be functionalized, such as } \\
\text { electron-rich arenes or electron-poor arenes. }\end{array}$ \\
\hline & High & $\begin{array}{l}\text { Several categories of arene substrates can be functionalized, such } \\
\text { as electron-rich arenes, electron-neutral arenes, as well as some } \\
\text { hetarenes. }\end{array}$ \\
\hline & Excellent & $\begin{array}{l}\text { Various categories of substrates including electron-rich, -neutral } \\
\text { and -poor arenes as well as various hetarenes can be successfully } \\
\text { converted. Many different substitution patterns are tolerated. }\end{array}$ \\
\hline
\end{tabular}


Conventional electrophilic aromatic nitration

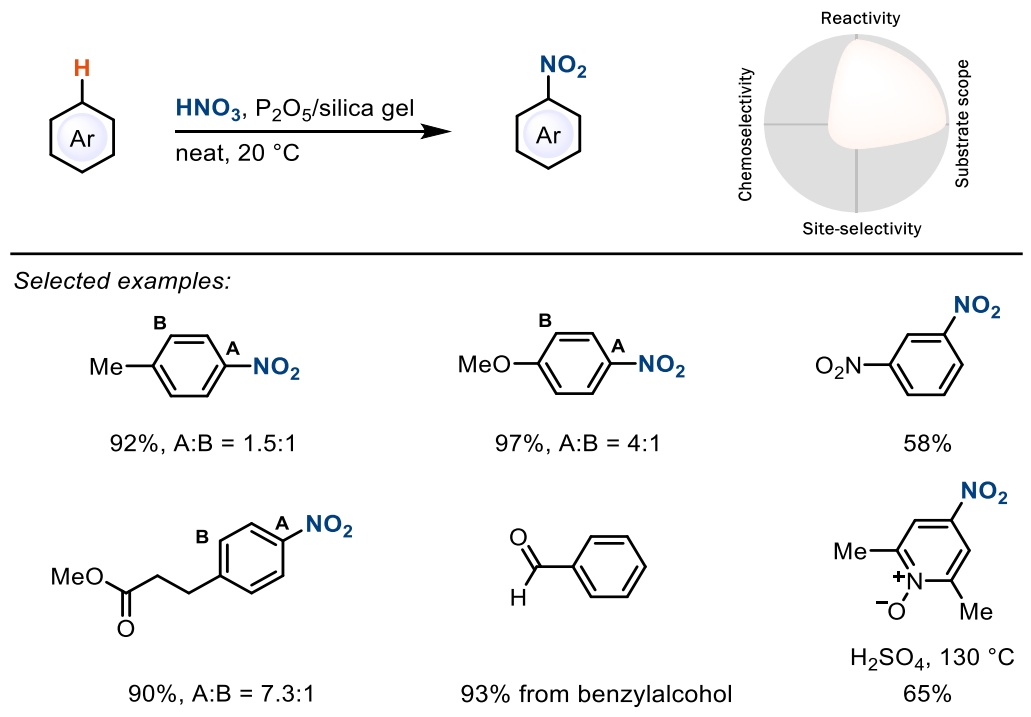

\begin{tabular}{|c|c|c|}
\hline Evaluation criteria & Score & Analysis ${ }^{1-3}$ \\
\hline Reactivity & Excellent & $\begin{array}{l}\text { 1) Reaction temperature is } 20^{\circ} \mathrm{C} \text { (or lower) for most substrates. }{ }^{1} \\
\text { 2) Electron-rich arenes are converted to the corresponding } \\
\text { nitroarenes in excellent yields within } 2-7 \mathrm{~min} .{ }^{1} \\
\text { 3) For electron-poor arenes, even nitrobenzene, the reaction is } \\
\text { complete within } 20 \mathrm{~min} \text { at } 20^{\circ} \mathrm{C} .{ }^{1}\end{array}$ \\
\hline Chemoselectivity & Low & $\begin{array}{l}\text { 1) When benzylalcohol was applied as substrate, alcohol oxidation } \\
\text { is observed (benzaldehyde, } 93 \% \text { yield). }{ }^{1} \\
\text { 2) Nitration of complex molecules under conventional conditions is } \\
\text { rare. } .^{1,2}\end{array}$ \\
\hline Site-selectivity & Low & $\begin{array}{l}\text { 1) The site-selectivity for toluene is } p: 0=1.5: 1 .^{1} \\
\text { 2) The site-selectivity for anisole is } p: 0=4: 1.1^{1} \\
\text { 3) Constitutional isomers are not observed in the nitration reaction } \\
\text { of nitrobenzene. }{ }^{1}\end{array}$ \\
\hline Substrate scope & Excellent & $\begin{array}{l}\text { 1) Electron-rich, -neutral or -poor arenes can be functionalized. }{ }^{1} \\
\text { 2) Electron-poor hetarenes such as pyridines or pyrimidines are } \\
\text { challenging substrates for electrophilic addition process, yet } \\
\text { nitration can proceed successfully on such electron-poor } \\
\text { hetarenes. }{ }^{3}\end{array}$ \\
\hline
\end{tabular}


Electrophilic aromatic bromination with a sulfide catalyst

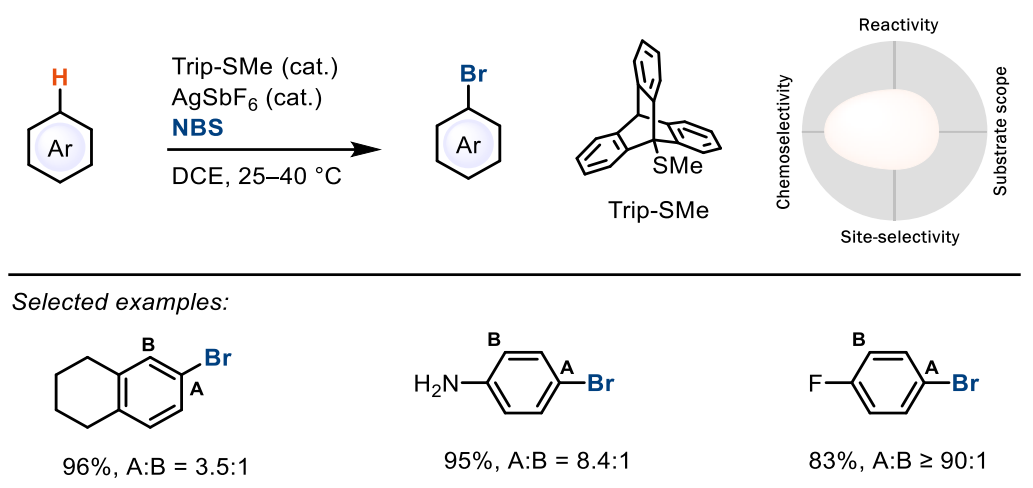<smiles>Oc1ccc2cc(Br)ccc2c1-c1c(O)ccc2cc(Br)ccc12</smiles>

$92 \%$

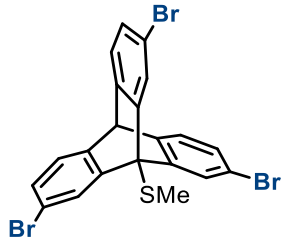

$53 \%$

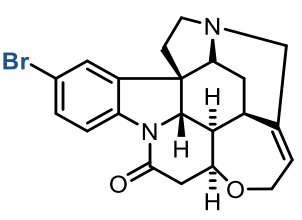

$96 \%$

\begin{tabular}{|c|c|c|}
\hline Evaluation criteria & Score & Analysis ${ }^{4-6}$ \\
\hline Reactivity & Moderate & $\begin{array}{l}\text { 1) Most reactions are complete within } 24 \text { hours at } 25^{\circ} \mathrm{C} \text {. } \\
\text { 2) The reaction requires } 5 \text { mol\% Trip-SMe catalyst. } \\
\text { 3) Bromination of the catalyst was observed, and the } \\
\text { brominated catalyst can cause a significant drop in yield } \\
\text { from } 82 \% \text { to } 18 \% \text { for } p \text {-xylene. } .^{4,5} \\
\text { 4) Elevated temperature }\left(40^{\circ} \mathrm{C} \text { ) or longer time }(24-46 \mathrm{~h}) \text { are }\right. \\
\text { necessary for electron-neutral arenes. } .^{4}\end{array}$ \\
\hline Chemoselectivity & High & $\begin{array}{l}\text { 1) Traditonal aromatic bromination catalyzed by } \mathrm{FeCl}_{3} \text { usually } \\
\text { suffer from bromination at benzylic positions, }{ }^{6} \text { which was not } \\
\text { observed in the sulfide-catalyzed bromination reaction. } \\
\text { 2) Substrates with amines or phenols can be functionalized } \\
\text { without protecting groups. }{ }^{4} \\
\text { 3) The sulfide-catalyzed bromination can be applied to } \\
\text { complex molecules containing a range of functional groups } \\
\text { such as amides, esters, epoxides, amines, phenols, } \\
\text { haologens, trifluoromethyls or hetarenes. }{ }^{4,5}\end{array}$ \\
\hline Site-selectivity & Moderate & $\begin{array}{l}\text { 1) The site-selectivity for } 1,2,3,4 \text {-tetrahydronaphthalene is } \\
\mathrm{C} 2: \mathrm{C} 1=3.5: 1.4 \\
\text { 2) The site-selectivity for aniline is } p: 0=8.4: 1.4 \\
\text { 3) The site-selectivity for fluorobenzene is } p: 0 \geq 90: 1 .{ }^{4}\end{array}$ \\
\hline Substrate scope & Moderate & $\begin{array}{l}\text { 1) Both electron-rich and -neutral arenes can be } \\
\text { functionalized. } 4,5 \\
\text { 2) Electron-rich hetarenes such as indole, furan or thiophene } \\
\text { can be functionalized. }{ }^{4} \\
\text { 3) Electron-deficient arenes such as methylbenzoate are not } \\
\text { reported as substrates for the electrophilic bromination } \\
\text { reaction. } .^{4,5}\end{array}$ \\
\hline
\end{tabular}




\section{Electrophilic aromatic fluorination via a $\left[\mathrm{Pd}^{\mathrm{IV}}\right]-\mathrm{F}$ species}

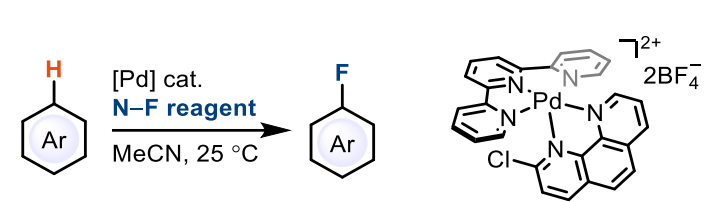

[Pd] cat.

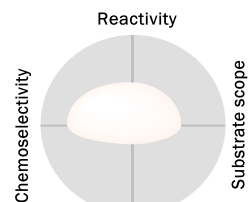

Site-selectivity

Selected examples:<smiles>Fc1ccc(Cl)cc1</smiles>

at $50{ }^{\circ} \mathrm{C}$

$49 \%, A: B=59: 41$

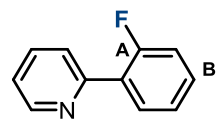

at $50{ }^{\circ} \mathrm{C}$ $55 \%, A: B=77: 23$

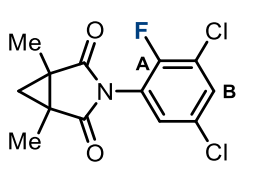

at $80^{\circ} \mathrm{C}$

$35 \%, A: B=56: 44$<smiles>CN1CC(C)(C)N=C1c1cn(-c2ccc(F)cc2)cc1N=O</smiles>

$54 \%$<smiles>O=C1CC2CCC(C1)N2S(=O)(=O)c1ccc(-c2ccccc2F)cc1</smiles>

$59 \%, A: B=71: 30$<smiles>Cc1cc(C(=O)N2CCCC(O)c3cc(Cl)ccc32)c(F)cc1NC(=O)c1ccccc1C</smiles>

at $50{ }^{\circ} \mathrm{C}$

$56 \%, A: B=51: 49$

\begin{tabular}{|c|c|c|}
\hline Evaluation criteria & Score & Analysis ${ }^{7-9}$ \\
\hline Reactivity & Moderate & $\begin{array}{l}\text { 1) For most substrates with an electron-donating group, the } \\
\text { reactions are complete within } 24 \mathrm{~h} \text { at } 25^{\circ} \mathrm{C} . .^{7} \\
\text { 2) Elevated temperature }\left(50-80^{\circ} \mathrm{C}\right) \text { or longer time }(25-36 \mathrm{~h}) \\
\text { are necessary for electron-neutral arenes, chlorobenzene or } \\
\text { complex molecules. }{ }^{7} \\
\text { 3) The catalyst loading is } 5-10 \text { mol\% for most substrates. }{ }^{7}\end{array}$ \\
\hline Chemoselectivity & High & $\begin{array}{l}\text { 1) Substrates with alcohol groups can be functionalized without } \\
\text { protection. } \\
\text { 2) Complex molecules containing ketones, esters, amides, } \\
\text { halogens, sulfonamides, alcohols or hetarenes can be } \\
\text { fluorinated. }\end{array}$ \\
\hline Site-selectivity & Low & $\begin{array}{l}\text { 1) The site-selectivity for chlorobenzene is } p: 0=59: 41 .{ }^{7} \\
\text { 2) The site-selectivity for } 2 \text {-phenylpyridine is } p: 0=77: 23 \text { on the } \\
\text { phenyl ring. }\end{array}$ \\
\hline Substrate scope & Moderate & $\begin{array}{l}\text { 1) Both electron-rich and -neutral arenes can be functionalized. }{ }^{7} \\
\text { 2) Electron-deficient arenes such as methylbenzoate are not } \\
\text { reported as substrates for the fluorination reaction. } \\
\text { 3) Hetarenes were not reported as substrates for the fluorination } \\
\text { reaction. }\end{array}$ \\
\hline
\end{tabular}


Electrophilic aromatic oxygenation with bis(methanesulfonyl) peroxide
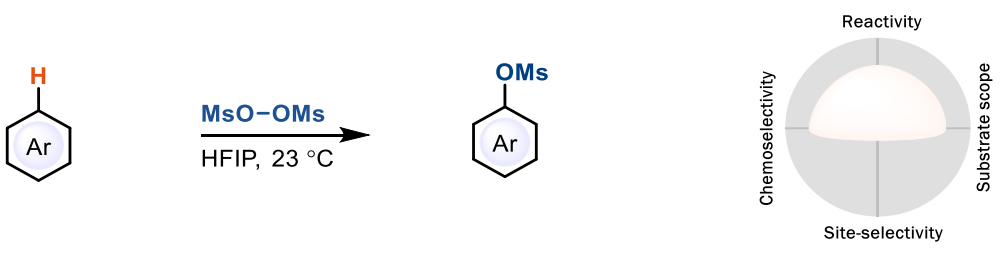<smiles>COC(=O)C(Cc1ccc(OC)cc1)NC(C)=O</smiles>

$56 \%, A: B: C=4: 2: 1$<smiles>COC(=O)C(C)c1ccc(-c2ccc(OC)cc2)c(F)c1</smiles>

$77 \%, A: B: C=4: 3: 2$<smiles>COC(=O)c1ccc(OC)cc1</smiles>

with $\left[\mathrm{Ru}(\mathrm{bpy})_{3}\right]\left(\mathrm{PF}_{6}\right)_{2}$ in $\mathrm{MeCN}$

$62 \%, A: B: C=2: 1: 1$<smiles>Cc1ccccc1C(=O)Nc1ccc(C(=O)N2CCCC(O)c3cc(Cl)ccc32)c(C)c1F</smiles>

$42 \%, A: B=1: 1$

\begin{tabular}{|c|c|c|}
\hline Evaluation criteria & Score & Analysis ${ }^{10-12}$ \\
\hline Reactivity & High & $\begin{array}{l}\text { 1) For most electron-rich and }- \text { neutral arenes, the reactions are } \\
\text { complete within } 12-24 \mathrm{~h} \text { at } 23^{\circ} \mathrm{C} . .^{10} \\
\text { 2) In some cases, low temperature }\left(0^{\circ} \mathrm{C}\right) \text { is necessary when } \\
\text { adding the reagent }(\mathrm{MsO})_{2} .^{10} \\
\text { 3) Single-electron-transfer }(\mathrm{SET}) \text { catalyst }\left[\mathrm{Ru}(\mathrm{bpy})_{3}\right]\left(\mathrm{PF}_{6}\right)_{2} \\
(2.5 \mathrm{~mol} \%) \text { is necessary for electron-deficient arenes. } \\
\text { 4) For most substrates, } 1.1-1.3 \text { equivalents of }(\mathrm{MsO})_{2} \text { is required to } \\
\text { achieve high conversion. }\end{array}$ \\
\hline Chemoselectivity & High & $\begin{array}{l}\text { 1) Oxygenation of arenes usually suffer from overoxidation } \\
\text { because the product phenol is easily oxidized, }{ }^{11} \text { which is not the } \\
\text { case here. }{ }^{12} \\
\text { 2) Substrates with alcohol groups can be functionalized without } \\
\text { protection. }{ }^{10} \\
\text { 3) Complex molecules containing amides, esters, sulfonamides, } \\
\text { alcohols, trifluoromethyls, and hetarenes can be functionalized. }{ }^{10}\end{array}$ \\
\hline Site-selectivity & Low & $\begin{array}{l}\text { 1) The site-selectivity for a protected amino acid, Ac-Phe-OMe, is } \\
p: 0: m=4: 2: 1.10 \\
\text { 2) The site-selectivity for methylbenzoate is } p: 0: m=2: 1: 1 .^{10}\end{array}$ \\
\hline Substrate scope & High & $\begin{array}{l}\text { 1) Both electron-rich and -neutral arenes can be functionalized. }{ }^{10} \\
\text { 2) A single-electron-transfer (SET) catalyst is necessary for } \\
\text { electron-poor arenes, yet oxygenation can proceed successfully } \\
\text { on electron-poor substrates such as methylbenzoate. }{ }^{10} \\
\text { 3) Hetarenes such as } 2,5 \text {-dichloropyridines, azoles, quinoxalines, } \\
\text { or thiophenes can be functionalized. }\end{array}$ \\
\hline
\end{tabular}


Aromatic $\mathrm{C}-\mathrm{H}$ bond functionalization via aryl thianthrenium salts

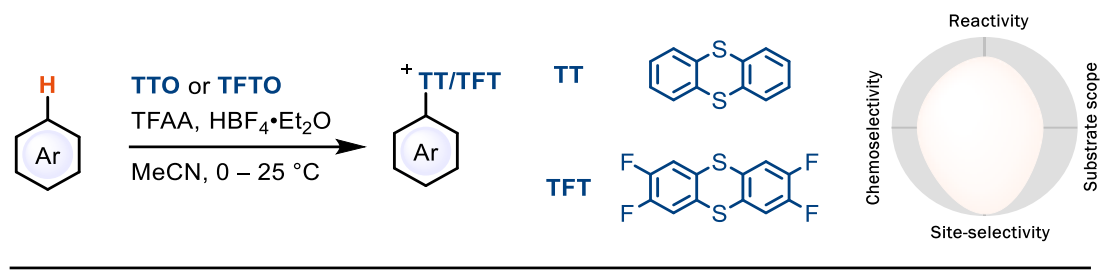

Selected examples:

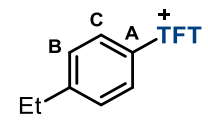

$92 \%, A: B>500: 1, A: C>200: 1$<smiles>CCc1ccc(C(C)(C)N)cc1COCc1cccc(Oc2ccccc2)c1</smiles>

$84 \%$<smiles></smiles>

$86 \%$<smiles>COc1ccc([In+])cn1</smiles>

$87 \%$<smiles>Cc1nc(Nc2ncc(C(=O)Nc3cc([N+](=O)[O-])cc(Cl)c3Cl)s2)cc(N2CCN(CCO)CC2)n1</smiles>

$80 \%$

\begin{tabular}{|c|c|c|}
\hline Evaluation criteria & Score & Analysis $^{13-15}$ \\
\hline Reactivity & High & $\begin{array}{l}\text { 1) For most substrates, the reactions are complete within } 1-24 \mathrm{~h} \\
\text { after warming from } 0^{\circ} \mathrm{C} \text { to } 25^{\circ} \mathrm{C} .^{13} \\
\text { 2) For chlorobenzene, the thianthrenation reaction is complete } \\
\text { within } 3 \mathrm{~h} \text { at } 0-25^{\circ} \mathrm{C} . .^{13} \\
\text { 3) High conversion of arenes can be achieved with } 1.0 \text { equivalents } \\
\text { of thianthrene-S-oxide. } .^{13}\end{array}$ \\
\hline Chemoselectivity & High & $\begin{array}{l}\text { 1) The key intermediates thianthrene dication }{ }^{14} \text { and thianthrene } \\
\text { radical cation }{ }^{15} \text { are not reported to undergo hydrogen atom } \\
\text { abstration, which is a common side reaction via electrophilic } \\
\text { aromatic substitution mechanism. } \\
\text { 2) Substrates with amines, phenols, alcohols or carboxylic acids } \\
\text { can be functionalized without protecting groups. }{ }^{13} \\
\text { 3) Complex molecules bearing amides, esters, sulfonamides, } \\
\text { alcohols, trifluoromethyls, and hetarenes can be } \\
\text { thianthrenated. }{ }^{13}\end{array}$ \\
\hline Site-selectivity & Excellent & $\begin{array}{l}\text { 1) The site-selectivity for ethylbenzene is } p: 0>500: 1 \text {, } \\
p: m>200: 1 .^{13} \\
\text { 2) Constitutional isomers were not observed in the thianthrenation } \\
\text { reaction of chlorobenzene. } .^{13} \\
\text { 3) High site-selectivity is achieved in almost all cases, even for } \\
\text { compounds containing several reactive positions. }{ }^{13}\end{array}$ \\
\hline Substrate scope & High & $\begin{array}{l}\text { 1) Both electron-rich and -neutral arenes can be functionalized. }{ }^{13} \\
\text { 2) Electron-deficient arenes such as chlorobenzene can be } \\
\text { functionalized, but more electron-deficient substrates such as } \\
\text { methylbenzoate are not reported as substrates for the } \\
\text { thianthrenation reaction. } .^{13} \\
\text { 3) Electron-rich hetarenes such as azoles, (benzo)furans, } \\
\text { (benzo)thiophenes or 2-methoxypyridine can be } \\
\text { thianthrenated. } .^{13}\end{array}$ \\
\hline
\end{tabular}




\section{Enzyme-catalyzed $\mathrm{C}-\mathrm{H}$ bond functionalization}
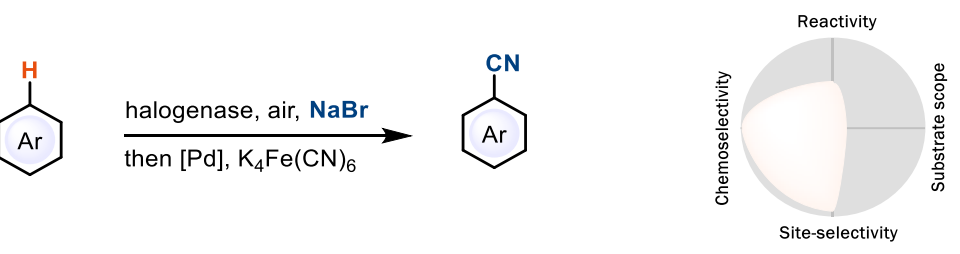

Selected examples:<smiles>N#Cc1ccc(N2CCOCC2)cc1</smiles><smiles>N#Cc1ccc2[nH]c3c(c2c1)CCNC3</smiles><smiles>N#Cc1ccc2c(CCC(=O)O)c[nH]c2c1</smiles><smiles>N#Cc1ccc(N2CCCN(CCC(O)c3csc4ccccc34)CC2)cc1</smiles><smiles>N#Cc1ccc(N2CCN(Cc3cc(C(=O)O)c(=O)n4ccccc34)CC2)cc1</smiles>

\begin{tabular}{|c|c|c|}
\hline Evaluation criteria & Score & Analysis $^{16-18}$ \\
\hline Reactivity & Moderate & $\begin{array}{l}\text { 1. Most reactions are complete within } 12 \mathrm{~h} \text { at } 25^{\circ} \mathrm{C} .{ }^{16} \\
\text { 2. For the natural substrates of halogenase, the conversion is high; } \\
\text { but the conversion is much lower when the structure of } \\
\text { substrates changes. For example, the conversion is } 97 \pm 4 \% \text { for } \\
\text { tryptophan but } 1.1 \pm 0.1 \% \text { for anthanilic acid with wild-type SttH. }{ }^{17}\end{array}$ \\
\hline Chemoselectivity & Excellent & $\begin{array}{l}\text { 1. Traditonal aromatic bromination reaction catalyzed by } \mathrm{FeCl}_{3} \\
\text { usually suffer from bromination reaction at benzylic position, }{ }^{6} \\
\text { which was not observed in the enzyme-catalyzed bromination } \\
\text { reaction. }{ }^{16} \\
\text { 2. Substrates with amines, carboxylic acids, alcohols, indoles, can } \\
\text { be functionalized without protecting groups. }{ }^{16} \\
\text { 3. Complex molecules bearing amides, amines, alcohols, and } \\
\text { hetarenes can undergo the bromination reaction smoothly. }{ }^{16}\end{array}$ \\
\hline Site-selectivity & Excellent & $\begin{array}{l}\text { 1. The site-selectivity is higly-dependent on the mutants used, the } \\
\text { constitutional isomers are not observed when a proper mutant of } \\
\text { the wild type enzyme was applied. }{ }^{16} \\
\text { 2. Site-selectivity can be controlled by the variation of different } \\
\text { halogenase, for example trytophan halogenation at C5, C6, or } \\
\text { C7 can be achieved with enzyme PyrH, SttH or PrnA } \\
\text { respectively. }{ }^{16-18}\end{array}$ \\
\hline Substrate scope & Low & $\begin{array}{l}\text { 1. The natural substrates of the enzyme can be halogenated } \\
\text { efficiently. }{ }^{17,18} \\
\text { 2. Aniline, phenol, pyrole or indole derivatives can be halogenated } \\
\text { with different mutants of flavin-dependent halogenases. }{ }^{16,17} \\
\text { 3. Substituted phenols or polycyclic arenes can be halogenated } \\
\text { with different mutants of heme-iron-dependent } \\
\text { haloperoxidases. }{ }^{18} \\
\text { 4. Electron-deficient arenes were not reported as substrates for the } \\
\text { enzyme-catalyzed electrophilic bromination reaction. } .^{16-18}\end{array}$ \\
\hline
\end{tabular}


Vicarious nucleophilic substitution

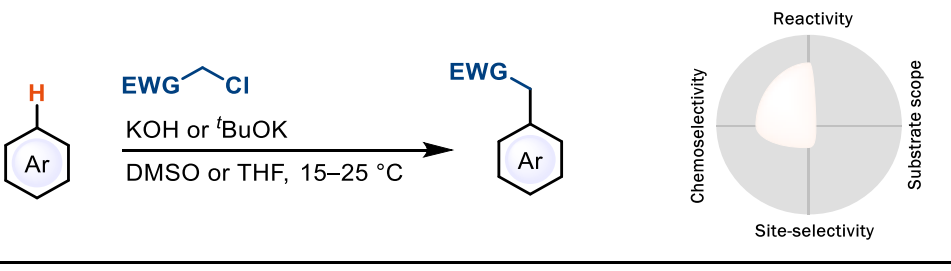

Selected examples:<smiles>C=CC=[C+]C=C(CS(=O)(=O)c1ccccc1)[N+](=O)[O-]</smiles>

$75 \%, A: B=53: 47$<smiles>O=[N+]([O-])C1=CC(CS(=O)(=O)c2ccccc2)C=C(C(F)(F)F)C1</smiles>

$67 \%, A: B=1: 1$

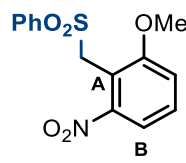

$42 \%, A: B=85: 15$<smiles>COc1nsc2c([N+](=O)[O-])c([N+](=O)[O-])cc([N+](=O)[O-])c12</smiles>

$90 \%$<smiles>O=[N+]([O-])c1cccc(Cl)c1CS(=O)(=O)c1ccccc1</smiles>

$93 \%, A: B: C=6: 73: 21$<smiles>O=S(=O)(Cc1cnncn1)c1ccnnc1</smiles>

$72 \%$

\begin{tabular}{|c|c|c|}
\hline Evaluation criteria & Score & Analysis ${ }^{19-21}$ \\
\hline Reactivity & High & $\begin{array}{l}\text { 1. Most reactions are complete within } 2 \mathrm{~h} \text { at } 15-25^{\circ} \mathrm{C} . .^{20-21} \\
\text { 2. Lower temperature }\left(-30^{\circ} \mathrm{C}\right) \text { is also applicable to avoid undesired } \\
\text { addition reactions at other electrophilic sites. } .^{21} \\
\text { 3. High conversion can be achieved with } 1.0 \text { equivalents of } \\
\text { nucleophiles. }{ }^{19-21}\end{array}$ \\
\hline Chemoselectivity & Moderate & $\begin{array}{l}\text { 1. Addition of the carbanion to carbonyl groups will lead to Darzens } \\
\text { condenzation product. }{ }^{21} \\
\text { 2. Nucleophilic ipso attack to substituted nitrobenzenes via } \\
\text { conventional SNAr mechansms is a possible side reaction, but } \\
\text { when a reactive leaving group was attached to the nucleophile, } \\
\text { the side reaction can be avoided. }{ }^{20} \\
\text { 3. Substrates with halogens, nitriles, trifluoromethyls or hetarenes } \\
\text { can be functionalized. }{ }^{21}\end{array}$ \\
\hline Site-selectivity & Low & $\begin{array}{l}\text { 1. The site-selectivity for nitrobenzene is } 0: p=53: 47 . .^{21} \\
\text { 2. When the para position is blocked by substitutents, the VNS } \\
\text { reaction is ortho selective. }{ }^{21}\end{array}$ \\
\hline Substrate scope & Low & $\begin{array}{l}\text { 1. Nitrobenzene derivatives can undergo vicarious nucleophilic } \\
\text { substitution reactions. }{ }^{19-21} \\
\text { 2. Highly electrophilic heteroarenes which do not contain a nitro } \\
\text { group such as quinoline- } N \text {-oxide, quinoxlaline- } N \text {-oxide, } \\
\text { benzoxazole, benzothiazole, acridine or } 1,2,4 \text {-triazine can also } \\
\text { undergo the substitution reaction but others cannot. }{ }^{21}\end{array}$ \\
\hline
\end{tabular}


Aromatic $\mathrm{C}-\mathrm{H}$ bond functionalization via pyridyl phosphonium salts

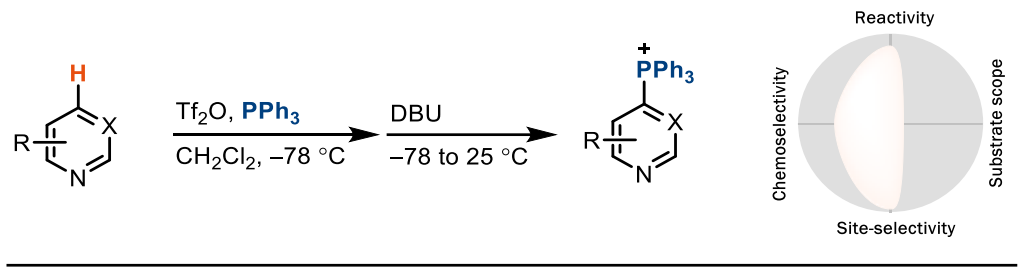

Selected examples:<smiles>Pc1cccc(P)c1</smiles>

$84 \%$<smiles>FC(F)(F)c1ccnc(P)c1</smiles>

$83 \%$<smiles>Cc1ccc(OCC2CCCN2Cc2cnccc2N(C)C)cn1</smiles>

DBU $65 \%, A: B>20: 1$

$\mathrm{Et}_{3} \mathrm{~N} 54 \%, \mathrm{~A}: \mathrm{B}<1: 20$

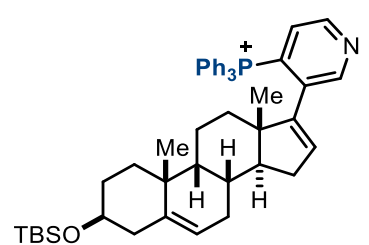

$74 \%$<smiles>C=CC1CC2CCC1C([C@H](O)c1cc([Pb])nc3ccccc13)C2</smiles>

$52 \%$<smiles>CCON1CCC(=C2c3ccc(Cl)cc3CCc3c([PH2+])ccnc32)CC1</smiles>

$88 \%$

\begin{tabular}{|c|c|c|}
\hline Evaluation criteria & Score & Analysis $22-24$ \\
\hline Reactivity & Excellent & $\begin{array}{l}\text { 1. Low temperature }\left(-78^{\circ} \mathrm{C}\right) \text { is necessary when adding triflic } \\
\text { anhydride, } \mathrm{PPh}_{3} \text {, and base, the reaction is complete within } 15- \\
30 \text { min after warming to } 25^{\circ} \mathrm{C} .^{22,23} \\
\text { 2. High conversion can be achieved when only } 1.0 \text { equivalent of } \\
\text { triflic anhydride and } 1.1 \text { equivalents of triarylphosphine are } \\
\text { applied. }{ }^{22,23}\end{array}$ \\
\hline Chemoselectivity & Moderate & $\begin{array}{l}\text { 1. Triflic anhydride must be applied to the substrate and may react } \\
\text { with several functional groups such as aldehydes, ketones, } \\
\text { alcohols etc. }{ }^{22} \\
\text { 2. Several substrates with secondary or tertiary amines can be } \\
\text { functionalized. }{ }^{22} \\
\text { 3. Complex molecules with amides, esters, trifluoromethyls, } \\
\text { halogens, alkynyls, alkenyls or hetarenes can be } \\
\text { functionalized. }{ }^{22}\end{array}$ \\
\hline Site-selectivity & Excellent & $\begin{array}{l}\text { 1. Only C4-substituted products can be observed when } 2-, \text { or } 3- \\
\text { phenyl pyridines were applied as substrates. When the C4 of } \\
\text { pyridines is substituted, the reaction is C2-selective. }{ }^{22} \\
\text { 2. Constitutional isomers cannot be observed for most pyridine } \\
\text { derivatives. }{ }^{22} \\
\text { 3. When more than one pyridine rings exist in the molecules, site- } \\
\text { selectivity can be controlled by base, phosphine, reagent order } \\
\text { or acyl-blocking strategies. }{ }^{24}\end{array}$ \\
\hline Substrate scope & Low & $\begin{array}{l}\text { 1. Pyridine derivatives can be functionalized. }{ }^{22,23} \\
\text { 2. Substituted diazines, quinolines or pyrimidines can be } \\
\text { functionalized.22,23 } \\
\text { 3. Functionalized arenes are not reported as substrates for the } \\
\text { transformation. }\end{array}$ \\
\hline
\end{tabular}


Functionalization of electron-rich (het)arenes via electrophilic metalation

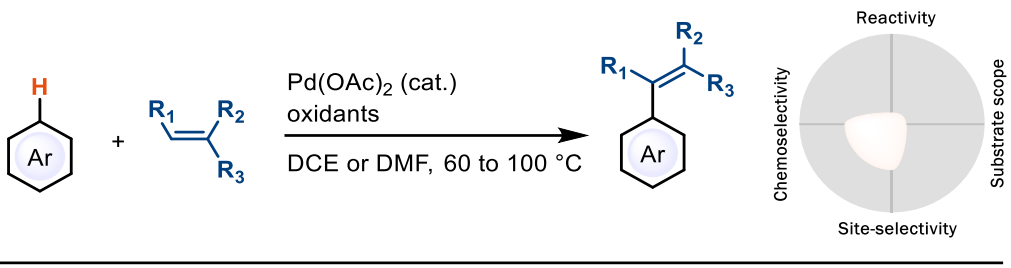

Selected examples:<smiles>COC(=O)C=Cc1cn(C)c2ccccc12</smiles>

$74 \%$<smiles>COC(=O)/C=C/c1ccc(N(C)[N+](=O)[O-])cc1</smiles>

$86 \%$<smiles>CCOC(=O)C=Cc1cn(C)c2cccc(C(=O)O)c12</smiles>

$63 \%$<smiles>COC(=O)/C=C/c1ccc(N(C)C(F)(F)F)cc1</smiles><smiles>CC(=O)Nc1cnc2cccc(Br)c2c1</smiles>

$87 \%$<smiles>CCOC(=O)C=C(c1ccccc1)c1ccc(OC)cc1</smiles>

\begin{tabular}{|c|c|c|}
\hline Evaluation criteria & Score & Analysis ${ }^{25-28}$ \\
\hline Reactivity & Low & $\begin{array}{l}\text { 1. For indole or aniline derivatives, most reactions are complete } \\
\text { within } 5-12 \text { hours at } 60-70^{\circ} \mathrm{C} . .^{25,26} \\
\text { 2. For anisole, a large excess of arene }(12-15 \text { equiv. }) \text {, high } \\
\text { pressure of oxygen }(8 \text { atm }) \text {, high temperature }\left(100^{\circ} \mathrm{C}\right) \text {, and long } \\
\text { reaction time }(25 \mathrm{~h}) \text { are necessary. } .^{27} \\
\text { 3. Stoichiometric amount of palladium acetate is required in the } \\
\text { olefination of some functionalized indole substrates. }{ }^{28}\end{array}$ \\
\hline Chemoselectivity & Moderate & $\begin{array}{l}\text { 1. Substrates with sulfonamides, amides, esters, ketones, } \\
\text { halogens, alkenes, nitriles, amines or hetarenes can be } \\
\text { functionalized. } .^{25,26} \\
\text { 2. Substrates with unprotected amines, alcohols or carboxylic acids } \\
\text { are not reported. } \\
\text { 3. The electrophilic metalation process has been applied to the } \\
\text { funtionalization of tryptophan derivatives and the total synthesis } \\
\text { of Clavicipitic acid. }{ }^{28}\end{array}$ \\
\hline Site-selectivity & Moderate & $\begin{array}{l}\text { 1. Constitutional isomers were not observed in the reactions of } \\
\text { most indole or aniline derivatives. } .^{25,26} \\
\text { 2. The site-selectivity for anisole is } p: 0: m=53: 32: 15 .{ }^{27}\end{array}$ \\
\hline Substrate scope & Low & $\begin{array}{l}\text { 1. Indole or aniline derivatives can undergo the electrophilic } \\
\text { metalation process followed by olefination reaction. }{ }^{25,26} \\
\text { 2. Other electron-rich or -neutral substrates show low reactivity. }{ }^{27} \\
\text { 3. Electron-deficient arenes were not reported as substrates for the } \\
\text { electrophilic metalation mechanism. }\end{array}$ \\
\hline
\end{tabular}


Palladium-catalyzed non-directed C-H bond olefination enabled by ligand

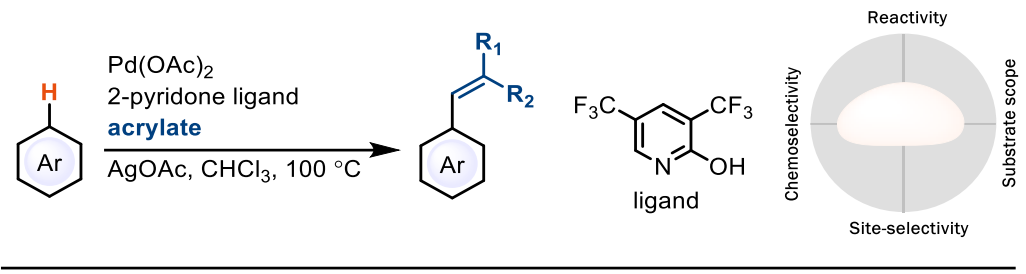

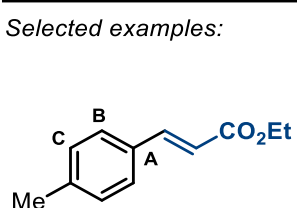

$83 \%, A: B: C=13.8: 12.2: 1$

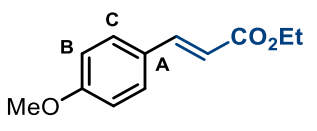

$67 \%, A: B: C=3.7: 1.8: 1$<smiles>CCOC(=O)C=Cc1cccc([N+](=O)[O-])c1</smiles>

$45 \%, A: B=14.1: 1$

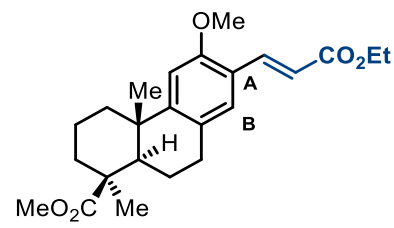

$58 \%, A: B=11.4: 1$<smiles>CCOC(C)=O</smiles>

$46 \%$

\begin{tabular}{|c|c|c|}
\hline Evaluation criteria & Score & Analysis $^{29-31}$ \\
\hline Reactivity & Moderate & $\begin{array}{l}\text { 1. Most reactions are complete within } 24 \text { hours at } 100^{\circ} \mathrm{C} . .^{29} \\
\text { 2. The limiting reagent is arene in the ligand-promoted } \\
\text { olefination. } 29-31 \\
\text { 3. The olefination reaction requires } 10 \mathrm{~mol} \% \mathrm{Pd}(\mathrm{OAc})_{2} \text { as well as } \\
30 \mathrm{~mol} \% 2 \text {-pyridone ligand. } 29\end{array}$ \\
\hline Chemoselectivity & High & $\begin{array}{l}\text { 1. Substrates with alcohols or carboxylic acids can be } \\
\text { functionalized without protecting groups. }{ }^{29} \\
\text { 2. Complex molecules containing sulfonamides, amides, esters, } \\
\text { halogens, trifluoromethyls, ketones, and hetarenes can be } \\
\text { functionalized. }{ }^{29}\end{array}$ \\
\hline Site-selectivity & Low & $\begin{array}{l}\text { 1. The site-selectivity for ethylbenzene is } m: p=1.0: 1 . .^{29} \\
\text { 2. The site-selectivity for anisole is } p: 0: m=3.7: 1.8: 1 .{ }^{29} \\
\text { 3. The site-selectivity for nitrobenzene is } m: p=14.1: 1 .{ }^{29} \\
\text { 4. Mono-olefinated products are the major products, and di- } \\
\text { olefinated products are formed as minor products when using } \\
\text { highly reactive susbtrates. }{ }^{29}\end{array}$ \\
\hline Substrate scope & High & $\begin{array}{l}\text { 1. Electron-rich and -poor arenes such as anisole, ethylbenzene, } \\
\text { methyl benzoate, and nitrobenzene are suitable susbtrates. }{ }^{29} \\
\text { 2. Hetarenes such as pyroles, carbazoles, (benzo)furans, } \\
\text { (benzo)thiophenes, and quinolines can be functionalized. }{ }^{29}\end{array}$ \\
\hline
\end{tabular}


Palladium-catalyzed non-directed C-H bond cyanation enabled by ligand

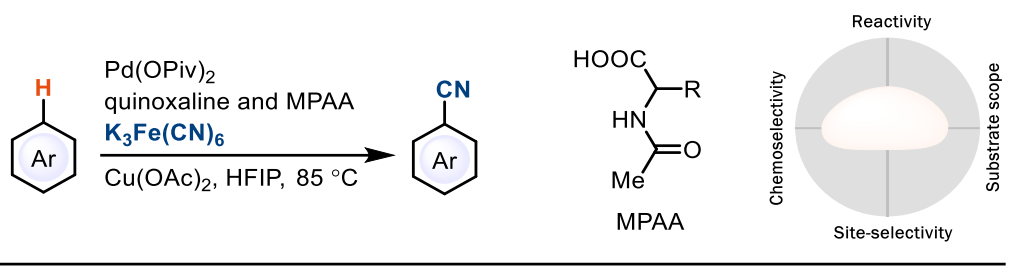

Selected examples:<smiles>Cc1cccc(C)c1</smiles>

$80 \%, A: B: C=6: 4: 1$<smiles>[N+]=[N+]=C1C=[C+]C=C1</smiles>

$92 \%, A: B=1: 1$<smiles>COc1cccc(C#N)c1</smiles>

$55 \%, A: B=3: 1$<smiles>N#Cc1cccc(-c2ccc(S(=O)(=O)N3CC4CC(=O)CC(C4)C3)cc2)c1</smiles>

$44 \%, A: B=1: 1$

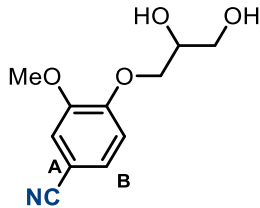

$50 \%, A: B=1: 1$

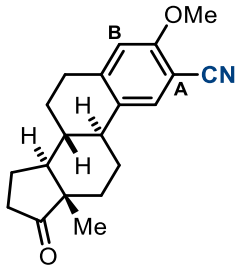

$87 \%, A: B=3: 1$

\begin{tabular}{|c|c|c|}
\hline Evaluation criteria & Score & Analysis $^{30,32}$ \\
\hline Reactivity & Moderate & $\begin{array}{l}\text { 1. Most reactions are complete within } 24 \text { hours at } 85^{\circ} \mathrm{C} .{ }^{32} \\
\text { 2. The limiting reagent is arene in the ligand-promoted cyanation. }{ }^{32} \\
\text { 3. The reaction requires } 10 \mathrm{~mol} \% \mathrm{Pd}(\mathrm{OPiv})_{2} \text { catalyst, and two kinds } \\
\text { of ligands including } 20 \mathrm{~mol} \% \mathrm{Ac}-\mathrm{Ala}-\mathrm{OH}^{30} \text { and } 30 \mathrm{~mol} \% \\
\text { quinoxaline. } .^{32}\end{array}$ \\
\hline Chemoselectivity & High & $\begin{array}{l}\text { 1. Substrates with alcohols, aldehydes or indoles can be } \\
\text { functionalized without protecting groups. }{ }^{32} \\
\text { 2. Complex molecules containing sulfonamides, amides, esters, } \\
\text { ketones, aldehydes, halogens, trifluoromethyls, and hetarenes } \\
\text { can be functionalized. }{ }^{32}\end{array}$ \\
\hline Site-selectivity & Low & $\begin{array}{l}\text { 1. The site-selectivity for toluene is } m: p: 0=6: 4: 1 .{ }^{32} \\
\text { 2. The site-selectivity for anisole is } 0: p=1: 1 .{ }^{2} \\
\text { 3. The site-selectivity for methyl benzoate is } m: p=3: 1 .{ }^{32}\end{array}$ \\
\hline Substrate scope & High & $\begin{array}{l}\text { 1. Electron-rich and -poor arenes such as anisole, ethylbenzene, } \\
\text { chlorobenzene, and methyl benzoate are successfully } \\
\text { cyanated. }{ }^{32} \\
\text { 2. Electron-rich hetarenes such as pyroles, indoles, thiophenes, } \\
\text { and } 2,5 \text {-dimethoxypyridine can be functionalized. } \text {. }^{32}\end{array}$ \\
\hline
\end{tabular}


Palladium-catalyzed ortho $\mathrm{C}-\mathrm{H}$ bond hydroxylation of aryl carboxylic acids

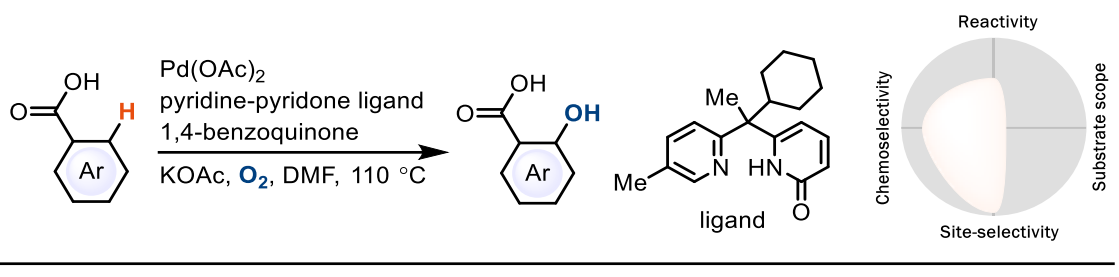<smiles>CC(C)(C)OC(=O)OC(=O)c1ccncc1O</smiles>

$72 \%$<smiles>O=C(O)c1cc(-c2ccccc2)ncc1O</smiles>

$66 \%$<smiles>O=C(O)c1ccc(-n2c(-c3ccccc3)cc3c2CCCC3=O)cc1O</smiles>

$80 \%$<smiles>CCCc1nc2c(C)cc(-c3nc4ccccc4n3C)cc2n1Cc1ccc(-c2cccc(O)c2C(=O)O)cc1</smiles><smiles>O=C(O)c1ccc(NC(=O)[C@H](Cc2ccc(O)cc2)NCc2ccccc2)cc1O</smiles>

$60 \%$

$71 \%$

\begin{tabular}{|c|c|c|}
\hline Evaluation criteria & Score & Analysis ${ }^{33,34}$ \\
\hline Reactivity & Moderate & $\begin{array}{l}\text { 1. Most reactions are complete within } 24 \text { hours at } 110^{\circ} \mathrm{C} .{ }^{33} \\
\text { 2. The reaction requires } 1 \text { atm of } \mathrm{O}_{2} .^{33} \text { Conventional oxygenation } \\
\text { without the pyridine-pyridone ligand requires high pressure of } \mathrm{O}_{2} \\
(5 \mathrm{~atm}) \text { to ensure sufficient reactivity. } .^{34} \\
\text { 3. The oxygenation reaction requires } 10 \mathrm{~mol} \% \mathrm{Pd}(\mathrm{OAc})_{2} \text { catalyst } \\
\text { and } 10 \mathrm{~mol} \% \text { pyridine-pyridone ligand. }{ }^{33}\end{array}$ \\
\hline Chemoselectivity & High & $\begin{array}{l}\text { 1. Substrates with phenols, amines, or carboxylic acids can be } \\
\text { functionalized without protecting groups. }{ }^{33} \\
\text { 2. Complex molecules containing halogens, amides, ketones, } \\
\text { amines, phenols, sulfonamides, carboxylic acids and hetarenes } \\
\text { can be functionalized. }{ }^{33}\end{array}$ \\
\hline Site-selectivity & Excellent & $\begin{array}{l}\text { 1. High site-selectivity is achieved in the presence of a range of } \\
\text { commonly used directing groups including -NHAc, -NHBoc, } \\
\text { aldehydes, and hetarenes. } \\
\text { 2. In the presence of one of the strongest directing groups for } \mathrm{C}-\mathrm{H} \\
\text { activation, 2-pyridyl group, the carboxyl group completely } \\
\text { overcomes the } 2 \text {-pyridyl-directing effect in the oxygenation } \\
\text { reaction. }{ }^{33} \\
\text { 3. High site-selectivity is observed in almost all cases. }\end{array}$ \\
\hline Substrate scope & Low & $\begin{array}{l}\text { 1. Aryl carboxylic acid derivatives with electron-rich or -poor arenes } \\
\text { can be functionalized. }{ }^{33} \\
\text { 2. Hetaryl carboxylic acids with benzothiophene, benzothiazole, } \\
\text { benzofuran, benzodioxane, morpholine, pyrrole, or carbazole } \\
\text { rings are successfully hydroxylated. }{ }^{33} \\
\text { 3. Arene substrates without carboxylic acid directing groups were } \\
\text { not reported as substrates. }\end{array}$ \\
\hline
\end{tabular}


Palladium-catalyzed remote $\mathrm{C}-\mathrm{H}$ bond olefination of quinoline via template

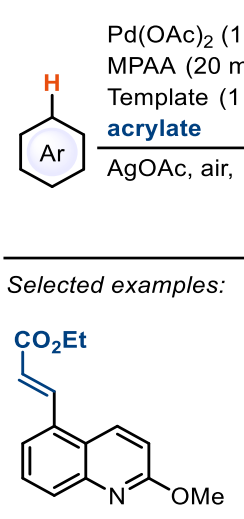

$67 \%$, C5:others $=84: 16$<smiles>CCOC(=O)C=Cc1cccc2ncsc12</smiles>

with another template $51 \%, C 7$ :others $=88: 12$
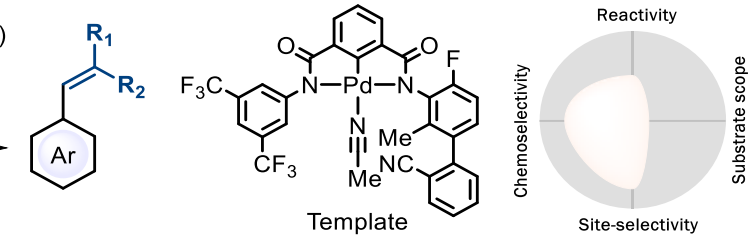

Site-selectivity<smiles>CCOC(=O)C=Cc1cccc2nc(Cl)ccc12</smiles>

$69 \%, \mathrm{C} 5:$ others $=85: 15$

$57 \%$, C $5:$ others $=88: 12$

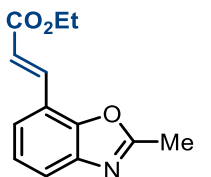

with another template $81 \%, \mathrm{C} 7$ : others $=87: 13$<smiles>CCOC(=O)CCc1cccc2cc3c(nc12)-c1cc2c(c(=O)n1C3)COC(=O)[C@@H]2O</smiles>

$49 \%$, C 5 :others $=89: 11$

\begin{tabular}{|c|c|c|}
\hline Evaluation criteria & Score & Analysis $^{35,36}$ \\
\hline Reactivity & Moderate & $\begin{array}{l}\text { 1. Most reactions are complete within } 48 \text { hours at } 80^{\circ} \mathrm{C} \text {. }^{35} \\
\text { 2. The remote olefination reaction requires } 10 \mathrm{~mol} \% \mathrm{Pd}(\mathrm{OAc})_{2} \text { and } \\
20 \mathrm{~mol} \% \text { Ac-Gly-OH ligand. }{ }^{35} \\
\text { 3. For quinoline substrates, } 1.0 \text { equivalent of template is required to } \\
\text { ensure the remote functionalization reactivity. }{ }^{35}\end{array}$ \\
\hline Chemoselectivity & High & $\begin{array}{l}\text { 1. Quinolines featuring esters, halogens, trifluoromethyls, amides, } \\
\text { or hetarenes can be functionalized via the template strategy. }{ }^{35} \\
\text { 2. Substrates with alcohol groups can be functionalized without } \\
\text { protecting groups. } \\
\text { 3. The remote } \mathrm{C}-\mathrm{H} \text { bond olefination can be applied to several } \\
\text { pharmaceuticals. }{ }^{35}\end{array}$ \\
\hline Site-selectivity & High & $\begin{array}{l}\text { 1. The site-selectivity for quinoline is C5:others }=90: 10 .{ }^{35} \\
\text { 2. The site-selectivity for benzothiazole is C7:others }=88: 12 .{ }^{35} \\
\text { 3. The site-selectivity for } 3 \text {-phenylpyridine is } m:(0+p)=95: 5 \text { on the } \\
\text { phenyl ring. }{ }^{35}\end{array}$ \\
\hline Substrate scope & Low & $\begin{array}{l}\text { 1. Quinoline, quinoxaline, benzoxazole, and benzothiazole } \\
\text { derivatives can be functionalized with different templates. }{ }^{35} \\
\text { 2. The template strategy is not feasible in the absence of an } \\
\text { appropriate coordinating functional group. }{ }^{36}\end{array}$ \\
\hline
\end{tabular}


Palladium-catalyzed remote $\mathrm{C}-\mathrm{H}$ bond arylation of quinoline via template and norbornene

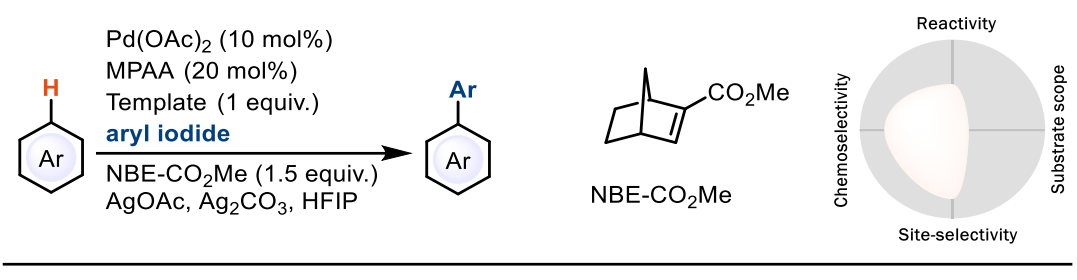

Selected examples:<smiles>COC(=O)c1ccccc1C(C)(Br)Br</smiles>

with another template $41 \%$

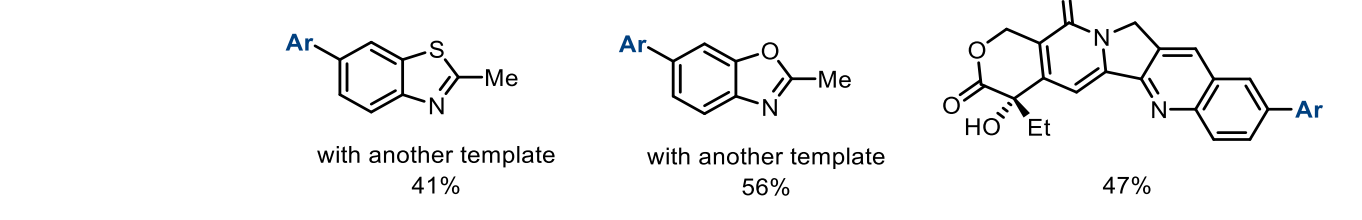<smiles>COc1ccc2cc(Br)ccc2n1</smiles>

$61 \%$<smiles>Cc1ccc2cc(Br)ccc2n1</smiles>

$70 \%$<smiles>Clc1ccc2cc(Br)ccc2n1</smiles>

$62 \%$

\begin{tabular}{|c|c|c|}
\hline Evaluation criteria & Score & Analysis $^{36,37}$ \\
\hline Reactivity & Moderate & $\begin{array}{l}\text { 1. Most reactions are complete within } 24 \text { hours at } 80^{\circ} \mathrm{C} .{ }^{37} \\
\text { 2. The remote arylation reaction requires } 10 \text { mol\% } \mathrm{Pd}(\mathrm{OAc})_{2} \text { as } \\
\text { well as } 20 \text { mol\% Ac-Gly-OH ligand. } .^{37} \\
\text { 3. For quinoline substrates, } 1.0 \text { equivalents of template is required } \\
\text { to ensure the remote functionalization reactivity. }{ }^{37}\end{array}$ \\
\hline Chemoselectivity & High & $\begin{array}{l}\text { 1. Quinolines featuring esters, halogens, trifluoromethyls, amides, } \\
\text { or hetarenes can be functionalized by cooperation of templates } \\
\text { and norbornene. }{ }^{37} \\
\text { 2. Substrates with alcohols can be functionalized without protecting } \\
\text { groups. }{ }^{37} \\
\text { 3. The remote } \mathrm{C}-\mathrm{H} \text { bond arylation can be applied to the } \\
\text { functionalization of several pharmaceuticals. }{ }^{37}\end{array}$ \\
\hline Site-selectivity & High & $\begin{array}{l}\text { 1. In the reaction of quinoline, no undesired consitutional isomer } \\
\text { was isolated. }{ }^{37} \\
\text { 2. In the reaction of } 3 \text {-methyl-isoquinoline, } 61 \% \text { of C5 } \\
\text { functionalization product as well as less than } 5 \% \text { over-arylated } \\
\text { product were observed. }{ }^{37}\end{array}$ \\
\hline Substrate scope & Low & $\begin{array}{l}\text { 1. Quinoline, isoquinoline, benzoxazole, benzothiophene, indazole, } \\
\text { or quinoxaline derivatives can be functionalized with different } \\
\text { templates. }{ }^{37} \\
\text { 2. The template and norbornene cooperation strategy is not } \\
\text { feasible in the absence of an appropriate coordinating functional } \\
\text { group. } .^{36}\end{array}$ \\
\hline
\end{tabular}


Ruthenium-catalyzed alkylation of $\mathrm{C}-\mathrm{H}$ bonds

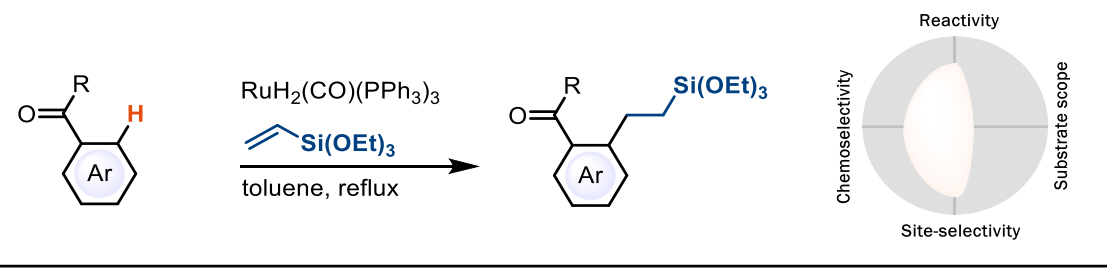

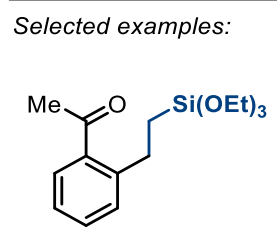

$75 \%$

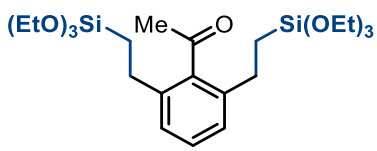

with 3 equiv. alkene

$94 \%$<smiles>CCO[SiH2]CCc1cccc(C(F)(F)F)c1C(=O)OC</smiles>

$97 \%$

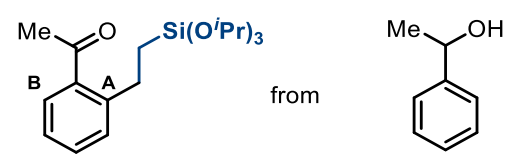

with $\mathrm{RuCl}_{3} \cdot \mathrm{HH}_{2} \mathrm{O}, \mathrm{P}\left(4-\mathrm{CF}_{3} \mathrm{C}_{6} \mathrm{H}_{4}\right)_{3}$ $96 \%$, mono: $\mathrm{di}=63: 37$

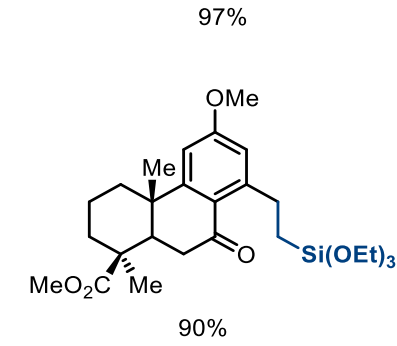

\begin{tabular}{|c|c|c|}
\hline Evaluation criteria & Score & Analysis ${ }^{38-41}$ \\
\hline Reactivity & High & $\begin{array}{l}\text { 1. The alkylation was performed in refluxing toluene (b.p. }=110^{\circ} \mathrm{C} \text { ) } \\
\text { in the seminal example, }{ }^{38} \text { but the reaction was complete in } 48 \mathrm{~h} \\
\text { at } 23^{\circ} \mathrm{C} \text { when a chelated ruthenium catalyst was applied. }{ }^{39} \\
\text { 2. The reaction requires } 2-6 \text { mol\% ruthenium catalyst. }{ }^{38,39} \\
\text { 3. Arenes are limiting reagents in the alkylation reaction, and the } \\
\text { reaction requires } 1-5 \text { equivalents of alkenes. } .^{38}\end{array}$ \\
\hline Chemoselectivity & Moderate & $\begin{array}{l}\text { 1. Aryl ketone substrates with esters, tertiary amines, } \\
\text { sulfonamides, silyl groups, or hetarenes can undergo the } \\
\text { alkylation reactions. } \\
\text { 2. When 1-phenylethanol was applied as substrate, alcohol } \\
\text { dehydrogenation is observed (alkylated ketone, } 96 \% \text { yield). }{ }^{39} \\
\text { 3. The ketone-directed C-H alkylation method can be employed to } \\
\text { derivatize diterpene natural products. }{ }^{40}\end{array}$ \\
\hline Site-selectivity & High & $\begin{array}{l}\text { 1. When acetophenone and } 2 \text { equivalents of triethoxyl(vinyl)silane } \\
\text { was applied as the substrates, the mono:di functionalization } \\
\text { selectivity changes from } 82: 18 \text { to } 98: 2 \text { when different ruthenium } \\
\text { catalysts were used. }{ }^{38,39} \\
\text { 2. When excess alkene was applied, di-alkylation species are the } \\
\text { major product. }{ }^{39}\end{array}$ \\
\hline Substrate scope & Low & $\begin{array}{l}\text { 1. Aryl ketone derivatives with both electron-rich and -poor arenes } \\
\text { can be functionalized. }{ }^{38,39} \\
\text { 2. Aryl esters and aldehydes can be successfully alkylated. }{ }^{39} \\
\text { 3. Ruthenium-catalyzed late-stage alkylation of arenes without } \\
\text { directing groups were not reported. }{ }^{41}\end{array}$ \\
\hline
\end{tabular}


Iridium-catalyzed borylation of $\mathrm{C}-\mathrm{H}$ bonds

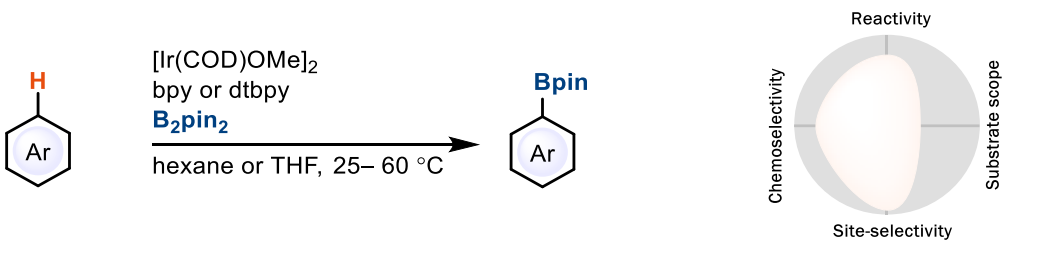

Selected examples:<smiles>COc1cccc(Br)c1</smiles>

$95 \%, A: B: C=74: 25: 1$<smiles>COc1cc(CC(Cc2ccccc2)Cc2ccccc2)cc(C(=O)OCc2ccccc2)c1</smiles>

$84 \%$, A:others $>98: 2$<smiles>[18OH]c1ccc(Cl)c(Cl)c1</smiles>

$83 \%$<smiles>CC(=O)CC(Cc1cc([18OH])cc(C(F)(F)F)c1)NC(C)=O</smiles>

$84 \%$, A:others $>98: 2$<smiles>COc1cc([18OH])cc([N+](=O)[O-])c1</smiles>

$72 \%$

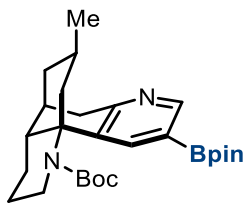

$75 \%$

\begin{tabular}{|c|c|c|}
\hline Evaluation criteria & Score & Analysis ${ }^{42-44}$ \\
\hline Reactivity & High & $\begin{array}{l}\text { 1. The original borylation reaction requires high temperature ( } 80 \\
\left.{ }^{\circ} \mathrm{C}\right) \text { and solvent amount of arene substrates }(60 \text { equiv.) in early } \\
\text { examples. }{ }^{42} \text { Modern versions use arene as the limiting reagent, } \\
\text { and the reactions are complete in } 24 \mathrm{~h} \text { at } 25^{\circ} \mathrm{C} .43 \\
\text { 2. Most reactions require } 3 \text { mol\% iridium catalyst, but the metal } \\
\text { catalyst can reach }>300 \text { turnovers for the functionalization of } \\
\text { benzene. }{ }^{43}\end{array}$ \\
\hline Chemoselectivity & High & $\begin{array}{l}\text { 1. The borylation can be applied to complex molecules containing } \\
\text { halogens, tertiary amines, trifluoromethyls, esters, nitriles, and } \\
\text { hetarenes. }{ }^{42-44} \\
\text { 2. Substrates with indoles, pyroles or ketones can be functionalized } \\
\text { without protecting groups }{ }^{42-44} \\
\text { 3. The iridium catalyzed C-H functionalization has been applied to } \\
\text { the total synthesis of several complex molecules such as (+)- } \\
\text { Complanadine A. }{ }^{44}\end{array}$ \\
\hline Site-selectivity & $\begin{array}{l}\text { Excellent } \\
\text { /Low }\end{array}$ & $\begin{array}{l}\text { 1. For 1,3-disubstituted arenes and several 1,2-disubstituted } \\
\text { arenes, the site-selectivity is excellent. For example, } \\
\text { constitutional isomers were not observed when 1,3- } \\
\text { dichlorobenzene was applied.43 } \\
\text { 2. For mono substituted arenes, the site-selectivity is low. For } \\
\text { examples, the site-selectivity for anisole is } m: p: 0=74: 25: 1 .{ }^{4}{ }^{4}\end{array}$ \\
\hline Substrate scope & $\begin{array}{l}\text { Low } \\
\text { /High }\end{array}$ & $\begin{array}{l}\text { 1. The substrate scope of the site-selective borylation low, } \\
\text { including symmetrically substituted } 1,2 \text { - and } 1,4 \text {-substituted } \\
\text { arenes, } 1,3 \text {-substituted arenes, benzothiophenes, benzofurans, } \\
\text { and indoles. } \\
\text { 2. The substrate scope of unselective borylation is high, including } \\
\text { but not limited to mono-substituted arenes with electron-donating } \\
\text { or -withdrawing groups. }{ }^{42,43}\end{array}$ \\
\hline
\end{tabular}


Iron-catalyzed hydrogen isotope exchange (HIE) reaction

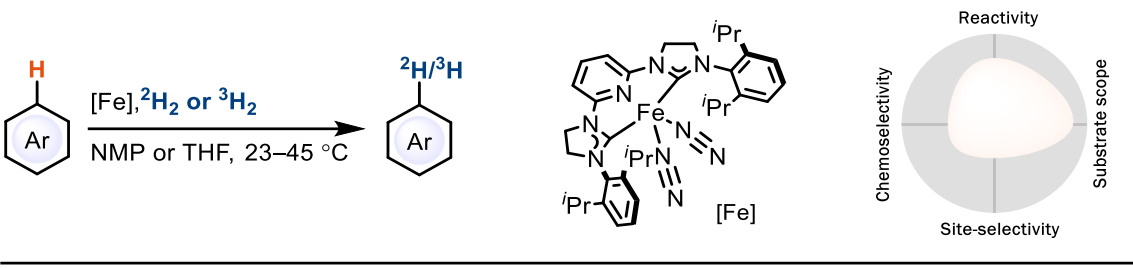

Selected examples:
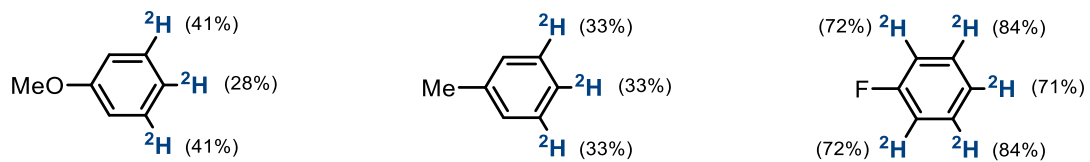

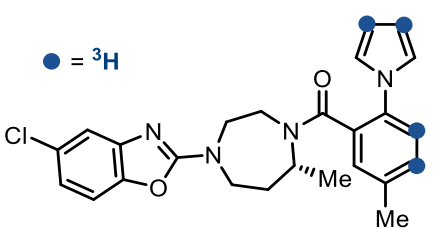

15.2 Ci mmo

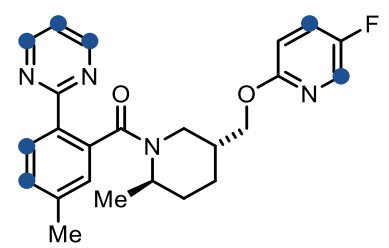

$57 \mathrm{Ci} \mathrm{mmol}$

\begin{tabular}{|c|c|c|}
\hline Evaluation criteria & Score & Analysis $^{45-47}$ \\
\hline Reactivity & High & $\begin{array}{l}\text { 1. Most reactions are complete within } 24 \mathrm{~h} \text { at } 23-45^{\circ} \mathrm{C} .45 \\
\text { 2. The deuteration reaction is carried out with } 1 \mathrm{~atm} \text { of } \\
\text { deuterium gas. The tritiation reaction can take place with low- } \\
\text { pressure }\left(120 \mathrm{mmHg} \text { ) of tritium gas. }{ }^{45}\right. \\
\text { 3. The loading of the iron catalyst is } 1 \mathrm{~mol} \% .{ }^{.45}\end{array}$ \\
\hline Chemoselectivity & Moderate & $\begin{array}{l}\text { 1. The HIE reaction can be applied to complex molecules } \\
\text { containing secondary and tertiary amines, halogens, } \\
\text { sulfonamides, amides, trifluoromethyls, esters, nitriles, and } \\
\text { hetarenes. }{ }^{45} \\
\text { 2. Substrates with carboxylic acids or amines can be } \\
\text { functionalized without protecting groups. }{ }^{45} \\
\text { 3. Substrates with ketones, aldehyde or alcohols are not } \\
\text { reported. }{ }^{45-47}\end{array}$ \\
\hline Site-selectivity & Low & $\begin{array}{l}\text { 1. The ratio of deuterium incorporation for toluene is } 33 \% \text { at the } \\
\text { meta position and } 33 \% \text { at the para position. } .5 \\
\text { 2. The ratio of deuterium incorporation for anisole is } 41 \% \text { at the } \\
\text { meta position and } 28 \% \text { at the para position. } 45 \\
\text { 3. The ratio of deuterium incorporation for trifluorobenzene is } \\
90 \% \text { at the meta position and } 90 \% \text { at the para position. } 45\end{array}$ \\
\hline Substrate scope & Excellent & $\begin{array}{l}\text { 1. Electron-rich and -poor arenes such as anisole, toluene, and } \\
\text { trifluorobenzene are all suitable substrates. }{ }^{45} \\
\text { 2. Electron-rich and -poor hetarenes such as pyrole, imidazole, } \\
\text { pyrazole, triazole, indole, thiophene, quinoline, and pyridine } \\
\text { can be functionalized. }{ }^{45}\end{array}$ \\
\hline
\end{tabular}


Oxygenation of arenes with phthaloyl peroxide
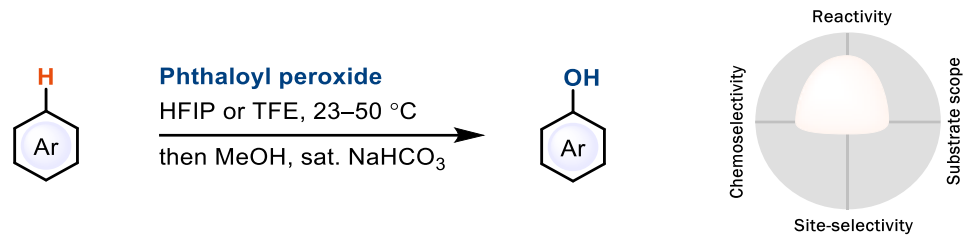

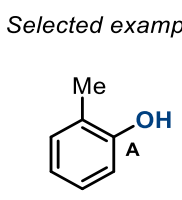

$46 \%, A: B=1: 1$<smiles>O=Cc1cccc(Oc2ccc(O)c(O)c2)c1</smiles>

$68 \%, A: B=1: 1$<smiles>COc1ccccc1O</smiles>

$B$

$45 \%, A: B=1.4: 1$

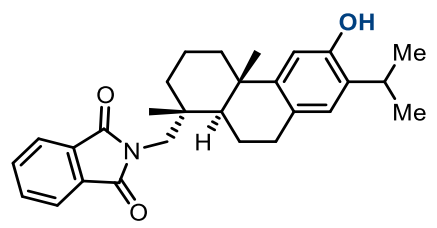

$63 \%$<smiles>Oc1ccccc1-c1ccccc1</smiles>

B

$65 \%, A: B=2.5: 1$

\begin{tabular}{|c|c|c|}
\hline Evaluation criteria & Score & Analysis $^{48}$ \\
\hline Reactivity & High & $\begin{array}{l}\text { 1. Most electron-rich arene substrates can be oxygenated at } 23^{\circ} \mathrm{C} \\
\text { within } 3-24 \mathrm{~h} .48 \\
\text { 2. Elevated temperature }\left(40-50{ }^{\circ} \mathrm{C}\right) \text { is required when electron- } \\
\text { neutral arene substrates are employed. } 48 \\
\text { 3. For most substrates, } 1.3 \text { equivalents of phthaloyl peroxide is } \\
\text { required to achieve high conversion. } .^{48}\end{array}$ \\
\hline Chemoselectivity & Moderate & $\begin{array}{l}\text { 1. Oxygenation with peroxides usually suffer from overoxidation or } \\
\text { benzylic oxidation reactions, }{ }^{11} \text { which were not observed in the } \\
\text { oxygenation reaction via phthaloyl peroxide. }{ }^{48} \\
\text { 2. Complex molecules containing esters, sulfonates, halogens, } \\
\text { trifluoromethyls, nitriles, aldehydes, epoxides, and hetarenes can } \\
\text { be functionalized. }{ }^{48} \\
\text { 3. Substrates with unprotected amines, alcohols or carboxylic acids } \\
\text { were not reported. }\end{array}$ \\
\hline Site-selectivity & Low & $\begin{array}{l}\text { 1. The site-selectivity for toluene is } o: p=1: 1.48 \\
\text { 2. The site-selectivity for } n \text {-butylbenzene is } o: p=1: 1.48 \\
\text { 3. The site-selectivity for anisole is } o: p=1.4: 1 .{ }^{48}\end{array}$ \\
\hline Substrate scope & Moderate & $\begin{array}{l}\text { 1. Both electron-rich and -neutral arenes can be functionalized. }{ }^{48} \\
\text { 2. Electron-deficient arenes such as chlorobenzene or } \\
\text { methylbenzoate are not reported as substrates for the } \\
\text { oxygenation reaction. } 48\end{array}$ \\
\hline
\end{tabular}


Palladium-catalyzed TEDAylation of arenes

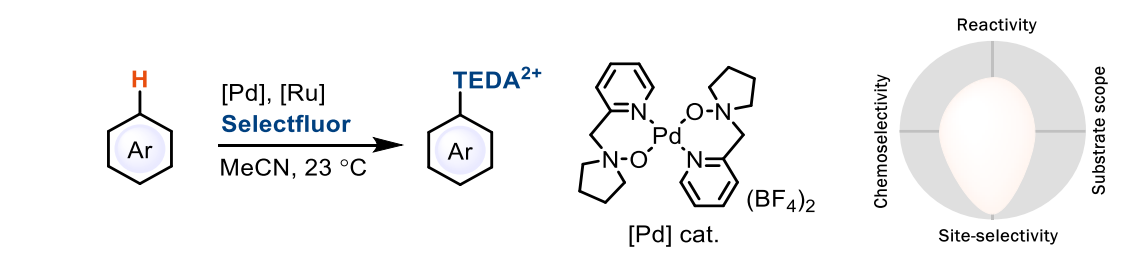

Selected examples:

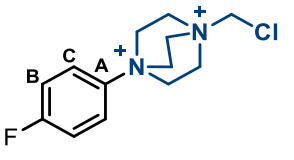

$88 \%$, A:others $>99: 1$<smiles>Cc1ccc(N2CCN(CCl)CC2)cc1</smiles>

$85 \%$<smiles>COc1ccc(N2CCN(CCl)CC2)cc1F</smiles>

$86 \%$<smiles>CC(C)OC(=O)C(C)(C)Oc1ccc(C(=O)c2ccc(Cl)cc2)cc1N1CCN(CCl)CC1</smiles>

$88 \%$<smiles>CC(=O)C(Cc1ccc(N2CCN(CCl)CC2)cc1)N1C(=O)c2ccccc2C1=O</smiles>

\begin{tabular}{|c|c|c|}
\hline Evaluation criteria & Score & Analysis ${ }^{49,50}$ \\
\hline Reactivity & Moderate & $\begin{array}{l}\text { 1. The TEDAylation reaction requires } 2.5 \mathrm{~mol} \% \text { palladium catalyst } \\
\text { and } 7.5 \mathrm{~mol} \%\left[\mathrm{Ru}(\mathrm{bpy})_{3}\right]\left(\mathrm{PF}_{6}\right)_{2} .49 \\
\text { 2. Most reactions are complete within } 24 \mathrm{~h} \text { at } 23{ }^{\circ} \mathrm{C} \text { for electron-rich } \\
\text { arene substrates. } 4^{49} \\
\text { 3. Elevated temperature }\left(40-45{ }^{\circ} \mathrm{C}\right) \text { is necessary for electron-poor } \\
\text { arenes such as chlorobenzene. } 49\end{array}$ \\
\hline Chemoselectivity & Moderate & $\begin{array}{l}\text { 1. It was reported that TEDA }{ }^{*+} \text { can cleave } s p^{3} \mathrm{C}-\mathrm{H} \text { bonds, however, } \\
\text { no such side reactions were reported in arene TEDAylation } \\
\text { reaction. } 49 \\
\text { 2. The TEDAylation reaction can be applied to molecules } \\
\text { containing amides, esters, ketones, trifluoromethyls, } \\
\text { sulfonamides, halogens, and hetarenes. }{ }^{49,50} \\
\text { 3. Substrates with unprotected amines, alcohols or carboxylic acids } \\
\text { were not reported. }\end{array}$ \\
\hline Site-selectivity & Excellent & $\begin{array}{l}\text { 1. The site-selectivity for fluorobenzene is } p: 0>99: 1.49 \\
\text { 2. Constitutional isomers were not observed in the TEDAylation } \\
\text { reaction of toluene. }{ }^{49} \\
\text { 3. When the para-position of arenes is substituted, the reaction is } \\
\text { ortho-selective. }{ }^{49,50}\end{array}$ \\
\hline Substrate scope & Moderate & $\begin{array}{l}\text { 1. Both electron-rich and -neutral arenes can be functionalized. } 49 \\
\text { 2. Electron-deficient arenes such as methylbenzoate were not } \\
\text { reported as substrates for the TEDAylation reaction. } 49 \\
\text { 3. Hetarenes such as thiophenes, quinolines, and } 2- \\
\text { methoxypyridine are successfully functionalized. } 49,50\end{array}$ \\
\hline
\end{tabular}


Electrochemical trifluoromethylation of hetarene with zinc sulfinate reagents

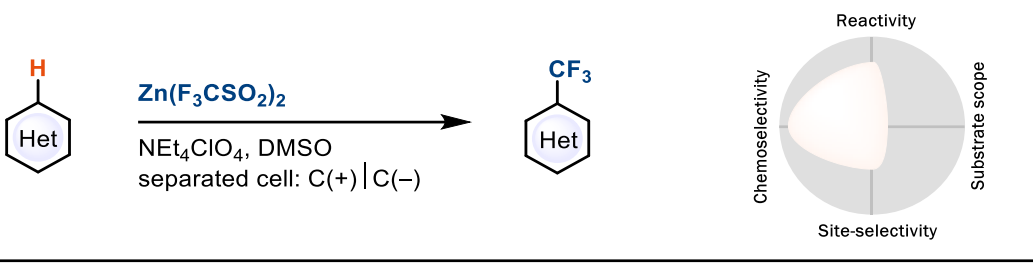

Selected examples:<smiles></smiles>

$53 \%, A: B=91: 9$

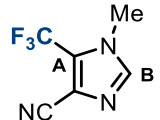

$40 \%, A: B=89: 11$<smiles>COC(=O)c1c(C(F)(F)F)n[13cH]n1C</smiles>

$38 \%, A: B=1: 1$<smiles>Cn1c(=O)c2c(nc(C(F)(F)F)n2C)n([O-])c1=O</smiles><smiles>Oc1nc2ccccc2nc1C(F)(F)F</smiles>

$\mathrm{H}_{2} \mathrm{~N}-L$ Q L P Y N S P V H S I- $-\mathrm{CO}_{2} \mathrm{H}$

$37 \%$

\begin{tabular}{|c|c|c|}
\hline Evaluation criteria & Score & Analysis $51-53$ \\
\hline Reactivity & High & $\begin{array}{l}\text { 1. Most of the reactions are complete in } 8 \mathrm{~h} \text { at } 25^{\circ} \mathrm{C} .5^{51} \\
\text { 2. A constant current of } 25 \mathrm{~mA} \text { is applied, and the cell potential } \\
\text { drifts from }+0.7 \mathrm{~V} \text { to }+1.3 \mathrm{~V} \text { during the reaction. } .^{51}\end{array}$ \\
\hline Chemoselectivity & Excellent & $\begin{array}{l}\text { 1. Electrochemical initiation avoids the use of peroxides. } \text {. }^{52,53} \\
\text { 2. Molecules containing esters, nitriles, nitros, halogens, alcohols, } \\
\text { trifluoromethyls, ketones, amines, and hetarenes can be } \\
\text { trifluoromethylated. }{ }^{51-53} \\
\text { 3. Substrates with alcohols, phenols or amines can be } \\
\text { functionalized without protecting groups. }{ }^{51} \\
\text { 4. The functionalization reaction can tolerate all } 20 \text { natural amino } \\
\text { acid residues, except free cysteine. } .^{53}\end{array}$ \\
\hline Site-selectivity & Moderate & $\begin{array}{l}\text { 1. The site-selectivity for } 1 \text {-methyl-4-cyano-imidazole is } \\
\text { C5:C2 }=89: 11 . .^{51} \\
\text { 2. Constitutional isomers were not observed in the } \\
\text { trifluoromethylation reaction of } 1 \text {-methyl-pyrazole. }{ }^{51} \\
\text { 3. For most substituted pyrole derivates, only } \mathrm{C} 2 \text { functionalization } \\
\text { products were observed. }{ }^{51}\end{array}$ \\
\hline Substrate scope & Low & $\begin{array}{l}\text { 1. Electron-rich hetarenes such as pyrole, pyrazole, imidazole, } \\
\text { indole or benzothiazole derivatives can be trifluoromethylated by } \\
\text { the approach. }{ }^{51} \\
\text { 2. Electron-poor hetarenes such as diazine, quinoxaline or pyridine } \\
\text { derivatives can be functionalized. }{ }^{51-53} \\
\text { 3. Functionalized arenes were not reported as substrates for the } \\
\text { reaction. }\end{array}$ \\
\hline
\end{tabular}


Amination of tyrosine via photoredox catalysis
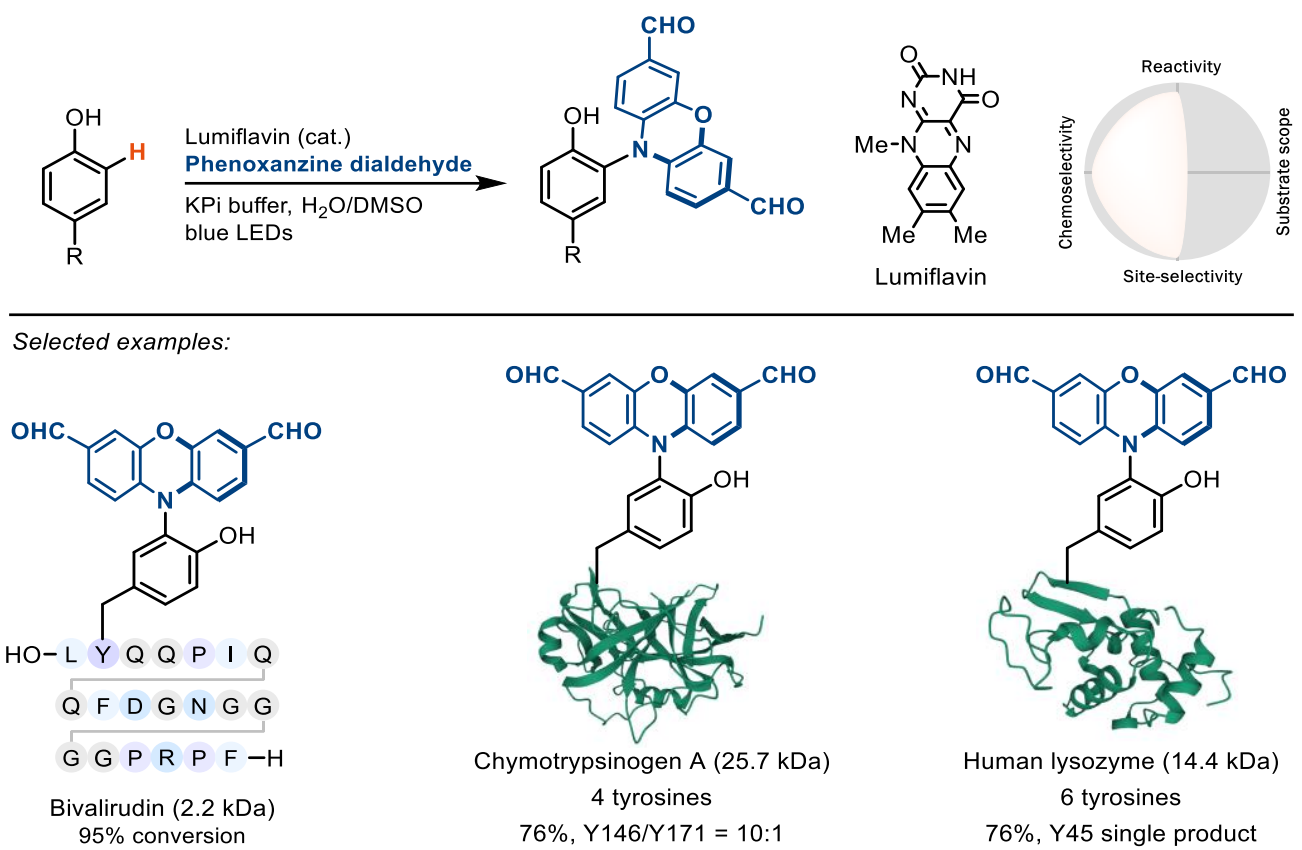

\begin{tabular}{|c|c|c|}
\hline Evaluation criteria & Score & Analysis $^{54,55}$ \\
\hline Reactivity & Excellent & $\begin{array}{l}\text { 1. Most reactions are complete within } 0.5-5 \mathrm{~h} \text { when irradiated } \\
\text { with a } 440 \mathrm{~nm} \text { photoreactor at } 4{ }^{\circ} \mathrm{C} .54 \\
\text { 2. The reaction was carried out with } 0.1 \mathrm{mM} \text { protein, } 0.3 \mathrm{mM} \\
\text { lumiflavin, and } 10 \mathrm{mM} \text { phenoxazine dialdehyde. } .^{54}\end{array}$ \\
\hline Chemoselectivity & Excellent & $\begin{array}{l}\text { 1. The amination reaction can tolerate all } 20 \text { natural amino acid } \\
\text { residues. }{ }^{54} \\
\text { 2. Proteins contain many phenols, alcohols, amines, indoles, } \\
\text { and carboxylic acids, but the reaction can take place without } \\
\text { protection. }{ }^{54}\end{array}$ \\
\hline Site-selectivity & Excellent & $\begin{array}{l}\text { 1. The bioconjugation reaction is ortho-selective with respect to } \\
\text { the phenol rings in tyrosine. } .^{54} \\
\text { 2. The bioconjugation reaction is tyrosine specific among } \\
\text { different amino acid residues. } .^{54} \\
\text { 3. The reaction is also selective between different tyrosine } \\
\text { residues on a protein. } .^{54}\end{array}$ \\
\hline Substrate scope & Low & $\begin{array}{l}\text { 1. Polypeptides and proteins with tyrosine residues can be } \\
\text { functionalized. } \text {. }^{54} \\
\text { 2. The photo-induced amination reaction on other residues was } \\
\text { not reported. }{ }^{55}\end{array}$ \\
\hline
\end{tabular}


Borylation of arenes with amine-borane

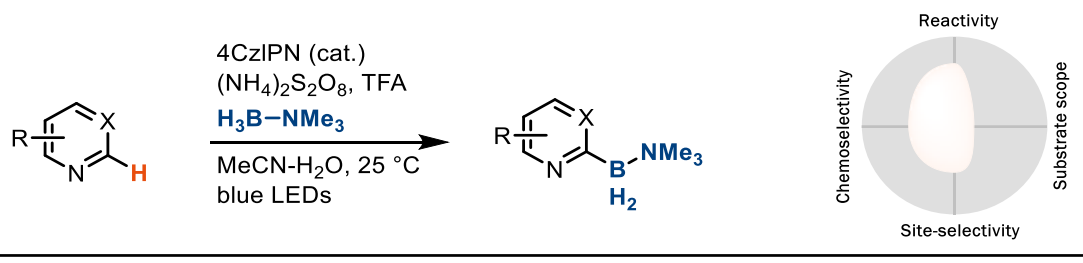

Selected examples:<smiles></smiles>

$63 \%, A: B=3: 1$<smiles></smiles>

$73 \%, A: B=2: 1$<smiles>CNc1nccc2ccccc12</smiles>

$57 \%$<smiles></smiles>

$35 \%$

\begin{tabular}{|c|c|c|}
\hline Evaluation criteria & Score & Analysis $^{56,57}$ \\
\hline Reactivity & High & $\begin{array}{l}\text { 1. The borylation reaction is complete within } 12 \mathrm{~h} \text { at } 25^{\circ} \mathrm{C} .{ }^{56} \\
\text { 2. The borylation reaction requires } 2 \text { mol\% photocatalyst and } \\
4.0 \text { equivalents of amino-borane to ensure high conversion. }{ }^{56} \\
\text { 3. The quantum yield of the radical borylation reaction is } \\
\Phi=0.41 . .^{56}\end{array}$ \\
\hline Chemoselectivity & Moderate & $\begin{array}{l}\text { 1. The borylation requires persulfate as the oxidant, which may } \\
\text { cause benzylic oxidation reactions. However, no such side } \\
\text { reactions were reported in the radical borylation. } .^{56} \\
\text { 2. The borylation reaction can be applied to complex molecules } \\
\text { containing amines, sulfonamides, halogens or hetarenes. }{ }^{56} \\
\text { 3. Substrates with phenols, alcohols or amines can be } \\
\text { functionalized without protecting groups. }{ }^{56} \\
\text { 4. Substrates with ketones or aldehydes were not reported. }{ }^{57}\end{array}$ \\
\hline Site-selectivity & Moderate & $\begin{array}{l}\text { 1. The site-selectivity for quinoline is } \mathrm{C} 2: \mathrm{C} 4=3: 1.56 \\
\text { 2. For isoquinoline, the borylation reaction is } \mathrm{C} 1 \text {-selective. }{ }^{56} \\
\text { 3. Only C2-substituted products can be observed when the } \mathrm{C} 4 \\
\text { position of pyridines or quinolines are substituted. } .^{56}\end{array}$ \\
\hline Substrate scope & Low & $\begin{array}{l}\text { 1. Pyridine, diazine, quinoline, isoquinoline, quinazoline, and } \\
\text { benzothiazole derivatives can be functionalized. } 56 \\
\text { 2. Functionalized arenes were not reported as substrates for the } \\
\text { transformation. }\end{array}$ \\
\hline
\end{tabular}


Radiofluorination reaction of aromatic $\mathrm{C}-\mathrm{H}$ bonds via photoredox catalysis

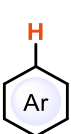

Mes-Acr ${ }^{+}$(cat.)
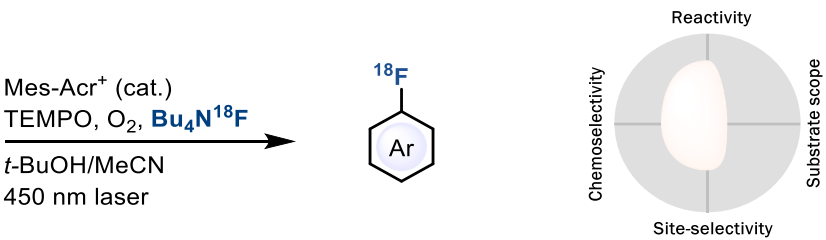

$450 \mathrm{~nm}$ laser

Site-selectivity

Selected examples:<smiles></smiles>

$38.2 \pm 10 \% \mathrm{RCY}$ for $\mathrm{A}$ $1.8 \pm 1 \% \mathrm{RCY}$ for $\mathrm{B}$<smiles>COc1ccc(F)c(C(N)=O)c1</smiles>

$14.8 \pm 0.6 \%$ RCY for $A$ $7.8 \pm 0.9 \%$ RCY for $B$

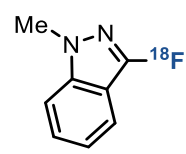

$14.4 \pm 3 \% \mathrm{RCY}$<smiles></smiles><smiles>COC(=O)c1cccc(Oc2ccc(F)cc2)c1</smiles>

$39.6 \pm 1 \% \mathrm{RCY}$

\begin{tabular}{|c|c|c|}
\hline Evaluation criteria & Score & Analysis 58,59 \\
\hline Reactivity & High & $\begin{array}{l}\text { 1. Most reactions are complete within } 30 \mathrm{~min} \text { at } 0{ }^{\circ} \mathrm{C} \text { when } \\
\text { irradiated with a } 450 \mathrm{~nm} \text { laser. } 58 \\
\text { 2. The reaction requires } 1 \mathrm{~atm} \text { of } \mathrm{O}_{2} .^{58} \\
\text { 3. The loading of photocatalyst Mes- } \mathrm{Acr}^{+} \mathrm{ClO}_{4}-\text { is } 1-5 \mathrm{~mol} \% .{ }^{58}\end{array}$ \\
\hline Chemoselectivity & Moderate & $\begin{array}{l}\text { 1. Complex molecules containing amides, sulfonates, halogens, } \\
\text { aldehydes, ketones, esters, and hetarenes can be } \\
\text { functionalized. }{ }^{58,59} \\
\text { 2. Substrates with unprotected amines, alcohols, carboxylic acids } \\
\text { were not reported. }{ }^{8}\end{array}$ \\
\hline Site-selectivity & Moderate & $\begin{array}{l}\text { 1. The site-selectivity for diphenyl ether is } p: 0=17.9: 1 \text { on the } \\
\text { phenyl ring. }{ }^{58} \\
\text { 2. The site-selectivity for 3-methoxyacetophenone is } \\
\text { C5:C2 }=1.9: 1.58 \\
\text { 3. Only ortho-substituted product was observed when the para } \\
\text { position of an electron-rich arene is substituted. }{ }^{58}\end{array}$ \\
\hline Substrate scope & Low & $\begin{array}{l}\text { 1. Electron-rich arenes such as anisole, indazole, benzimidazole, } \\
\text { and benzoxazole derivatives are successfully radiofluorinated. }{ }^{58} \\
\text { 2. Biphenyl or naphthalene derivatives can be functionalized. } .^{58} \\
\text { 3. Electron-neutral or -poor arenes are not reported as substrates } \\
\text { for the radiofluorination reaction. }\end{array}$ \\
\hline
\end{tabular}


Hydroxylation of aromatic C-H bonds via electrophotoredox catalysis
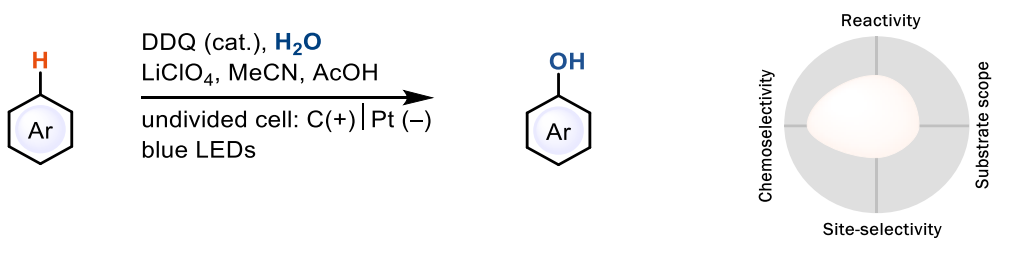

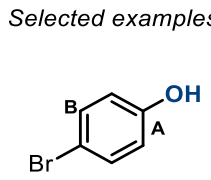

$55 \%, A: B=10: 1$<smiles>O=C(c1ccccc1)c1ccc(O)cc1</smiles>

$46 \%, A: B: C=3: 1: 1$<smiles>Oc1ccc(Cl)c(Cl)c1</smiles>

$41 \%$

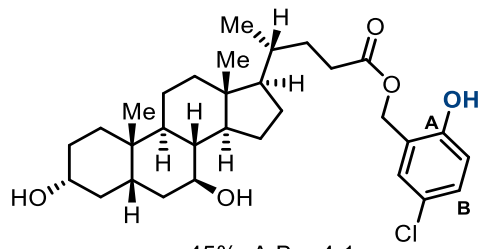<smiles>CC(=O)C(NC(=O)C(C)C)C(=O)OCc1cc(Cl)ccc1O</smiles>

$37 \%, A: B=3: 1$

\begin{tabular}{|c|c|c|}
\hline Evaluation criteria & Score & Analysis $^{60,61}$ \\
\hline Reactivity & Moderate & $\begin{array}{l}\text { 1. The oxygenation reaction requires } 10 \mathrm{~mol} \% \\
\text { electrophotocatalyst. }{ }^{60} \\
\text { 2. Most reactions are complete within } 12 \mathrm{~h} \text { under a controlled } \\
\text { potential of } 1.5 \mathrm{~V} \text { as well as irradiation from a blue LED at } \\
255^{\circ} \mathrm{C} .60\end{array}$ \\
\hline Chemoselectivity & High & $\begin{array}{l}\text { 1. Oxygenation of arenes usually suffer from overoxidation or } \\
\text { benzylic oxidation reactions, }{ }^{11} \text { which were not observed in the } \\
\text { reactions via electrophotoredox catalysis. }{ }^{60} \\
\text { 2. Complex molecules containing amides, sulfonamides, halogens, } \\
\text { ketones, esters, and alcohols can be oxygenated.60 } \\
\text { 3. Substrates with alcohols can be functionalized without protecting } \\
\text { groups. } 60\end{array}$ \\
\hline Site-selectivity & Moderate & $\begin{array}{l}\text { 1. The site-selectivity for bromobenzene is } p: 0=10: 1.60,61 \\
\text { 2. The site-selectivity for benzophenone is } p: 0: m=3: 1: 1.60 \\
\text { 3. Constitutional isomers are not observed in the oxygenation } \\
\text { reaction of } 1,2 \text {-dichlorobenzene. }{ }^{1}\end{array}$ \\
\hline Substrate scope & Moderate & $\begin{array}{l}\text { 1. Both electron-rich and -neutral arenes can be functionalized. } 60 \\
\text { 2. Electron-poor arenes such as bromobenzene can be } \\
\text { functionalized, but more electron-poor substrates such as } \\
\text { methylbenzoate were not reported as substrates for the } \\
\text { oxygenation reaction. }{ }^{60}\end{array}$ \\
\hline
\end{tabular}




\section{REFERENCES}

1. Hajipour, A. R.; Ruoho, A. E. Nitric acid in the presence of $\mathrm{P}_{2} \mathrm{O}_{5}$ supported on silica gel-a useful reagent for nitration of aromatic compounds under solvent-free conditions. Tetrahedron Lett. 2005, 46, 8307-8310.

2. Calvo, R.; Zhang, K.; Passera, A.; Katayev, D. Facile access to nitroarenes and nitroheteroarenes using $N$-nitrosaccharin. Nat. Commun. 2019, 10, 3410.

3. Zohuri, G. H.; Seyedi, S. M.; Sandaroos, R.; Damavandi, S.; Mohammadi, A. Novel late transition metal catalysts based on iron: synthesis, structures and ethylene polymerization. Catal. Lett. 2010, 140, 160-166.

4. Nishii, Y.; Ikeda, M.; Hayashi, Y.; Kawauchi, S.; Miura, M. Triptycenyl sulfide: A practical and active catalyst for electrophilic aromatic halogenation using N-halosuccinimides. J. Am. Chem. Soc. 2020, 142, 1621-1629.

5. Ueno, K.; Nishii, Y.; Miura, M. Electrophilic Substitution of Asymmetrically Distorted Benzenes within Triptycene Derivatives. Org. Lett. 2021, 23, 3552-3556.

6. Tanemura, K.; Suzuki, T.; Nishida, Y.; Satsumabayashi, K.; Horaguchi, T. Halogenation of Aromatic Compounds by $\mathrm{N}$-chloro-, $\mathrm{N}$-bromo-, and $\mathrm{N}$-iodosuccinimide. Chem. Lett. 2003, 32, 932-933.

7. Yamamoto, K.; Li, J.; Garber, J. A.; Rolfes, J. D.; Boursalian, G. B.; Borghs, J. C.; Genicot, C.; Jacq, J.; van Gastel, M.; Neese, F.; Ritter, T. Palladium-catalysed electrophilic aromatic C-H fluorination. Nature, 2018, 554, 511-514.

8. Taylor, S. D.; Kotoris, C. C.; Hum, G. Recent advances in electrophilic fluorination. Tetrahedron, 1999, 55, 12431-12477.

9. Lal, G. S.; Pez, G. P.; Syvret, R. G. Electrophilic NF fluorinating agents. Chem. Rev. 1996, 96, 1737-1756.

10. Börgel, J.; Tanwar, L.; Berger, F.; Ritter, T. Late-stage aromatic C-H oxygenation. J. Am. Chem. Soc. 2018, 140, 16026-16031.

11. Udenfriend, S.; Clark, C. T.; Axelrod, J.; Brodie, B. B. Ascorbic Acid In Aromatic Hydroxylation: I. A Model System for Aromatic Hydroxylation. J. Biol. Chem. 1954, 208, 731-740.

12. Stang, P. J.; Anderson, A. G. Hammett and Taft substituent constants for the mesylate, tosylate, and triflate groups. J. Org. Chem. 1976, 41, 781-785.

13. Berger, F.; Plutschack, M. B.; Riegger, J.; Yu, W.; Speicher, S.; Ho, M.; Frank, N.; Ritter, T. Site-selective and versatile aromatic $\mathrm{C}-\mathrm{H}$ functionalization by thianthrenation. Nature 2019, $567,223-228$.

14. Juliá, F.; Shao, Q.; Duan, M.; Plutschack, M. B.; Berger, F.; Mateos, J.; Lu, C.; Xue, X.-S.; Houk, K. N.; Ritter, T. High Site-Selectivity in Electrophilic Aromatic Substitutions: Mechanism of C-H Thianthrenation. J. Am. Chem. Soc. 2021, 143, 16041-16054.

15. Shine, H. J.; Silber, J. J. Ion radicals. XXII. Reaction of thianthrenium perchlorate $\left(\mathrm{C} 12 \mathrm{H} 8 \mathrm{S2}{ }^{+} \mathrm{ClO}^{-}\right)$with aromatics. J. Org. Chem. 1971, 36, 2923-2926.

16. Craven, E. J.; Latham, J.; Shepherd, S. A.; Khan, I.; Diaz-Rodriguez, A.; Greaney, M. F.; Micklefield, J. Programmable late-stage $\mathrm{C}-\mathrm{H}$ bond functionalization enabled by integration of enzymes with chemocatalysis. Nat. Catal. 2021, 4, 385-394.

17. Shepherd, S. A.; Menon, B. R.; Fisk, H.; Struck, A. W.; Levy, C.; Leys, D.; Micklefield, J. A structure-guided switch in the regioselectivity of a tryptophan halogenase. ChemBioChem 2016, 17, 821-824.

18. Latham, J.; Brandenburger, E.; Shepherd, S. A.; Menon, B. R.; Micklefield, J. Development of halogenase enzymes for use in synthesis. Chem. Rev. 2018, 118, 232-269. 
19. Makosza, M.; Winiarski, J. Vicarious nucleophilic substitution of hydrogen. Acc. Chem. Res. 1987, 20, 282-289.

20. Zlotin, S. G.; Kislitsin, P. G.; Kucherov, F. A.; Serebryakov, E. A.; Strelenko, Y. A.; Gakh, A. A. Synthetic utilization of polynitro aromatic compounds. 5. Multi-centered reactivity pattern in reactions of 4,6-dinitro-1,2-benzisothiazoles and -isothiazol-3(2H)-ones with C-, N-, O-, S, and F-nucleophiles. Heterocycles 2006, 68, 2483-2498.

21. Majkosza, M.; Danikiewicz, W.; Wojciechowski, K. Vicarious nucleophilic substitution with sulfur containing carbanions. Phosphorus Sulfur Silicon Relat. Elem. 1990, 53, 457-475.

22. Hilton, M. C.; Dolewski, R. D.; McNally, A. Selective functionalization of pyridines via heterocyclic phosphonium salts. J. Am. Chem. Soc. 2016, 138, 13806-13809.

23. Dolewski, R. D.; Hilton, M. C.; McNally, A. 4-Selective Pyridine Functionalization Reactions via Heterocyclic Phosphonium Salts. Synlett 2018, 29, 08-14.

24. Dolewski, R. D.; Fricke, P. J.; McNally, A. Site-selective switching strategies to functionalize polyazines. J. Am. Chem. Soc. 2018, 140, 8020-8026.

25. Wang, Y. J.; Yuan, C. H.; Chu, D. Z.; Jiao, L. Regiocontrol in the oxidative Heck reaction of indole by ligand-enabled switch of the regioselectivity-determining step. Chem. Sci. 2020, $11,11042-11054$.

26. Moghaddam, F. M.; Pourkaveh, R.; Karimi, A. Oxidative Heck Reaction as a Tool for Paraselective Olefination of Aniline: A DFT Supported Mechanism. J. Org. Chem. 2017, 82, 10635-10640.

27. Dams, M.; De Vos, D. E.; Celen, S.; Jacobs, P. A. Toward Waste-Free Production of Heck Products with a Catalytic Palladium System under Oxygen. Angew. Chem., Int. Ed. 2003, 42, 3512-3515.

28. Yokoyama, Y.; Matsumoto, T.; Murakami, Y. Optically active total synthesis of clavicipitic acid. J. Org. Chem. 1995, 60, 1486-1487.

29. Wang, P.; Verma, P.; Xia, G.; Shi, J.; Qiao, J. X.; Tao, S.; Cheng, P. T. W.; Poss. M. A.; Farmer, M. E.; Yeung, K.; Yu, J. Q. Ligand-accelerated non-directed C-H functionalization of arenes. Nature 2017, 551, 489-493.

30. Wang, D. H.; Engle, K. M.; Shi, B. F.; Yu, J. Q. Ligand-enabled reactivity and selectivity in a synthetically versatile aryl C-H olefination. Science 2010, 327, 315-319.

31. Lafrance, M.; Rowley, C. N.; Woo, T. K.; Fagnou, K. Catalytic intermolecular direct arylation of perfluorobenzenes. J. Am. Chem. Soc. 2006, 128, 8754-8756.

32. Zhao, D.; Xu, P.; Ritter, T. Palladium-catalyzed late-stage direct arene cyanation. Chem 2019, 5, 97-107.

33. Li, Z.; Wang, Z.; Chekshin, N.; Qian, S.; Qiao, J. X.; Cheng, P. T.; Yeung, K. S.; Ewing, W. $\mathrm{R}$.; Yu, J. Q. A tautomeric ligand enables directed $\mathrm{C}-\mathrm{H}$ hydroxylation with molecular oxygen. Science 2021, 372, 1452-1457.

34. Zhang, Y. H.; Yu, J. Q. Pd (II)-catalyzed hydroxylation of arenes with 1 atm of $\mathrm{O} 2$ or air. J. Am. Chem. Soc. 2009, 131, 14654-14655.

35. Zhang, Z.; Tanaka, K.; Yu, J. Q. Remote site-selective C-H activation directed by a catalytic bifunctional template. Nature 2017, 543, 538-542.

36. Meng, G.; Lam, N. Y.; Lucas, E. L.; Saint-Denis, T. G.; Verma, P.; Chekshin, N.; Yu, J. Q. Achieving site-selectivity for $\mathrm{C}-\mathrm{H}$ activation processes based on distance and geometry: a carpenter's approach. J. Am. Chem. Soc. 2020, 142, 10571-10591.

37. Shi, H.; Lu, Y.; Weng, J.; Bay, K. L.; Chen, X.; Tanaka, K.; Verma, P.; Houk, K.N.; Yu, J.Q. Differentiation and functionalization of remote $\mathrm{C}-\mathrm{H}$ bonds in adjacent positions. Nat. Chem. 2020, 12, 399-404. 
38. Murai, S.; Kakiuchi, F.; Sekine, S.; Tanaka, Y.; Kamatani, A.; Sonoda, M.; Chatani, N. Efficient catalytic addition of aromatic carbon-hydrogen bonds to olefins. Nature 1993, 366, 529-531.

39. Dong, Z.; Ren, Z.; Thompson, S. J.; Xu, Y.; Dong, G. Transition-metal-catalyzed C-H alkylation using alkenes. Chem. Rev. 2017, 117, 9333-9403.

40. (a) Harris, P. W. R.; Woodgate, P. D. Ruthenium-Catalysed Ortho Alkylation of Hydroxyacetophenones; the Functionalisation of Ring C Aromatic Diterpenoids. J. Organomet. Chem. 1996, 506, 339-341. (b) Harris, P. W. R.; Woodgate, P. D. RutheniumCatalysed Ortho Alkylation of Hydroxyacetophenones; the Functionalisation of Ring C Aromatic Diterpenoids. J. Organomet. Chem. 1997, 530, 211-223. (c) Wright, J. S.; Sharninghausen, L. S.; Preshlock, S.; Brooks, A. F.; Sanford, M. S.; Scott, P. J. Sequential Ir/Cu-Mediated Method for the Meta-Selective C-H Radiofluorination of (Hetero) Arenes. J. Am. Chem. Soc. 2021, 143, 6915-6921.

41. Lail, M.; Arrowood, B. N.; Gunnoe, T. B. Addition of arenes to ethylene and propene catalyzed by ruthenium. J. Am. Chem. Soc. 2003, 125, 7506-7507.

42. Ishiyama, T.; Takagi, J.; Ishida, K.; Miyaura, N.; Anastasi, N. R.; Hartwig, J. F. Mild iridiumcatalyzed borylation of arenes. High turnover numbers, room temperature reactions, and isolation of a potential intermediate. J. Am. Chem. Soc. 2002, 124, 390-391.

43. Hartwig, J. F. Borylation and silylation of $\mathrm{C}-\mathrm{H}$ bonds: a platform for diverse $\mathrm{C}-\mathrm{H}$ bond functionalizations. Acc. Chem. Res. 2012, 45, 864-873.

44. (a) Meyer, F. M.; Liras, S.; Guzman-Perez, A.; Perreault, C.; Bian, J.; James, K. Functionalization of Aromatic Amino Acids via Direct $\mathrm{C}-\mathrm{H}$ Activation: Generation of Versatile Building Blocks for Accessing Novel Peptide Space. Org. Lett. 2010, 12, 3870-3873.

(b) Fischer, D. F.; Sarpong, R. Total synthesis of (+)-complanadine A using an iridiumcatalyzed pyridine C- H functionalization. J. Am. Chem. Soc. 2010, 132, 5926-5927.

45. Yu, R. P.; Hesk, D.; Rivera, N.; Pelczer, I.; Chirik, P. J. Iron-catalysed tritiation of pharmaceuticals. Nature 2016, 529, 195-199.

46. Arevalo, R.; Chirik, P. J. Enabling two-electron pathways with iron and cobalt: from ligand design to catalytic applications. J. Am. Chem. Soc. 2019, 141, 9106-9123.

47. Corpas, J.; Viereck, P.; Chirik, P. J. C (sp2)-H Activation with Pyridine Dicarbene Iron Dialkyl Complexes: Hydrogen Isotope Exchange of Arenes Using Benzene-d 6 as a Deuterium Source. ACS Catal. 2020, 10, 8640-8647.

48. Yuan, C.; Liang, Y.; Hernandez, T.; Berriochoa, A.; Houk, K. N.; Siegel, D. Metal-free oxidation of aromatic carbon-hydrogen bonds through a reverse-rebound mechanism. Nature 2013, 499, 192-196.

49. Boursalian, G. B.; Ham, W. S.; Mazzotti, A. R.; Ritter, T. Charge-transfer-directed radical substitution enables para-selective C-H functionalization. Nat. Chem. 2016, 8, 810-815.

50. Serpier, F.; Pan, F.; Ham, W. S.; Jacq, J.; Genicot, C.; Ritter, T. Selective Methylation of Arenes: A Radical C-H Functionalization/Cross-Coupling Sequence. Angew. Chem., Int. Ed. 2018, 130, 10857-10861.

51. O'Brien, A. G.; Maruyama, A.; Inokuma, Y.; Fujita, M.; Baran, P. S.; Blackmond, D. G. Radical C-H Functionalization of Heteroarenes under Electrochemical Control. Angew. Chem., Int. Ed. 2014, 53, 11868-11871.

52. (a) Ji, Y.; Brueckl, T.; Baxter, R. D.; Fujiwara, Y.; Seiple, I. B.; Su, S.; Blackmond, D. G.; Baran, P. S. Innate C-H trifluoromethylation of heterocycles. Pro. Nat. Aca. Sci. 2011, 108, 14411-14415. (b) Fujiwara, Y.; Dixon, J. A.; O'Hara, F.; Funder, E. D.; Dixon, D. D.; Rodriguez, R. A.; Baxter, R. D.; Herle, B.; Sach, N.; Collins, M. R.; Ishihara, Y.; Baran, P. S. Practical and innate carbon-hydrogen functionalization of heterocycles. Nature 2012, 492, 95-99. 
53. Noisier, A. F.; Johansson, M. J.; Knerr, L.; Hayes, M. A.; Drury III, W. J.; Valeur, E.; Valeur, E.; Malins, L. R.; Gopalakrishnan, R. Late-Stage Functionalization of Histidine in Unprotected Peptides. Angew. Chem., Int. Ed. 2019, 131, 19272-19278.

54. For selected examples on tyrosine modification, see: (a) Song, C.; Liu, K.; Wang, Z.; Ding, B.; Wang, S.; Weng, Y.; Chiang, C-W; Lei, A. Electrochemical oxidation induced selective tyrosine bioconjugation for the modification of biomolecules. Chem. Sci. 2019, 10, 79827987. (b) Li, B. X.; Kim, D. K.; Bloom, S.; Huang, R. Y. C.; Qiao, J. X.; Ewing, W. R.; Oblinsky, D. G.; Scholes, G. D.; MacMillan, D. W. C. Site-selective tyrosine bioconjugation via photoredox catalysis for native-to-bioorthogonal protein transformation. Nat. Chem. 2021, 13, 902-908.

55. (a) Sletten, E. M.; Bertozzi, C. R. Bioorthogonal chemistry: fishing for selectivity in a sea of functionality. Angew. Chem., Int. Ed. 2009, 48, 6974-6998. (b) Tong, H. R.; Li, B.; Li, G.; He, G.; Chen, G. Postassembly modifications of peptides via metal-catalyzed C-H functionalization. CCS Chem. 2021, 3, 1797-1820.

56. Kim, J. H.; Constantin, T.; Simonetti, M.; Llaveria, J.; Sheikh, N. S.; Leonori, D. A radical approach for the selective C-H borylation of azines. Nature 2021, 595, 577-583.

57. Taniguchi, T. Boryl radical addition to multiple bonds in organic synthesis. Eur. J. Org. Chem. 2019, 2019, 6308-6319.

58. Chen, W.; Huang, Z.; Tay, N. E.; Giglio, B.; Wang, M.; Wang, H.; Wu, Z.; Nicewicz, D. A.; Li, Z. Direct arene $\mathrm{C}-\mathrm{H}$ fluorination with ${ }^{18} \mathrm{~F}^{-}$via organic photoredox catalysis. Science 2019, 364, 1170-1174.

59. Romero, N. A.; Margrey, K. A.; Tay, N. E.; Nicewicz, D. A. Site-selective arene CH amination via photoredox catalysis. Science 2015, 349, 1326-1330.

60. Huang, H.; Lambert, T. H. Electrophotocatalytic C-H Heterofunctionalization of Arenes. Angew. Chem., Int. Ed. 2021, 60, 11163-11167.

61. Ohkubo, K., Fujimoto, A.; Fukuzumi, S. Visible-light-induced oxygenation of benzene by the triplet excited state of 2, 3-dichloro-5, 6-dicyano-p-benzoquinone. J. Am. Chem. Soc. 2013, $135,5368-5371$. 